\title{
UK Renal Registry 11th Annual Report (December 2008): Chapter 4 ESRD prevalent rates in 2007 in the UK: national and centre-specific analyses
}

\author{
Ken Farrington ${ }^{\mathrm{a}}$, Alex Hodsman ${ }^{\mathrm{b}}$, Anna Casula ${ }^{\mathrm{b}}$, David Ansell ${ }^{\mathrm{b}}$ and John Feehally ${ }^{\mathrm{c}}$ \\ aLister Hospital, Stevenage, UK; ${ }^{b}$ UK Renal Registry, Bristol, UK; ' Leicester General Hospital, Leicester, UK
}

\section{Key Words}

Comorbidity - Diabetes - Dialysis - End Stage Renal Disease End Stage Renal Failure - Ethnicity - Haemodialysis - Peritoneal dialysis . Prevalence - Primary Care Trust - Renal replacement therapy · Transplantation · Treatment modality

\begin{abstract}
Introduction: This chapter describes the demographics of UK RRT patients in 2007. Methods: Complete data were electronically collected from $71 \mathrm{UK}$ centres with the remaining 1 centre submitting summary data. A series of crosssectional and longitudinal analyses were performed to describe the demographics of prevalent UK RRT patients in 2007 at a centre and a national level. Results: There were 45,484 adult patients receiving RRT on 31/12/2007. The population prevalence for adults was 746 per million population per year (pmp) with an annual increase in prevalence of approximately $5 \%$ per annum. There was substantial variation in standardised prevalence ratios between Primary Care Trust (PCT)/Health Authority (HA) areas which were associated with geographical factors and differences in ethnicity with mean standardised prevalence ratios (SPR) significantly higher in PCTs/HAs with a high proportion of ethnic minorities. The median age of prevalent RRT patients was 57 years (HD 65 years, PD 60 years, transplant 50 years). Median RRT vintage was 5.3 years (HD 2.8 years, PD 2.1 years, transplant 10.4 years). For all ages, crude prevalence rates in males exceeded
\end{abstract}

\section{KARGER}

Fax +4161306 1234 E-Mail karger@karger.ch www.karger.com
(C) $2009 \mathrm{~S}$. Karger AG, Basel

$1160-2110 / 09 / 1115-0043 \$ 26.00 / 0$

Accessible online at: www.karger.com/nec those in females, peaking in the 75-79 year age band for males at 2,506 pmp and in females in the 70-74 year age band at 1,314 pmp. The most common identifiable diagnosis was glomerulonephritis (15.3\%) but in those over 65 it was diabetes (15.1\%). The most common treatment modality was transplantation (46.6\%), closely followed by centrebased $\mathrm{HD}(42.1 \%)$ in either the primary centre $(25.2 \%)$ or the satellite unit (16.9\%). The HD population has continued to expand, and the PD population to contract. HD was increasingly prominent with increasing age at the expense of transplantation. Conclusions: There were national, area and dialysis centre level variation in the prevalent UK RRT population. This has implications for service planning and ensuring equity of care for RRT patients.

\section{Introduction}

The UK Renal Registry collected data from 72 (100\%) UK renal centres. Seventy one centres submitted an electronic dataset and one centre submitted summary data including prevalent patient numbers.

These analyses of prevalent RRT patients are performed annually in conjunction with a similar analysis of incident patients to aid clinicians and policy makers in planning future RRT requirements in the UK. It is important to understand national, regional and centre level variation in numbers of prevalent patients as part

Ken Farrington

UK Renal Registry, Southmead Hospital, Southmead Road, Bristol BS10 5NB, UK

Email: ken.farrington@nhs.net 
of this planning process. In addition, variation in case mix is also reported to improve understanding of where resources should be focussed to improve equity of provision of RRT in the UK.

The term Established Renal Failure (ERF) used within this chapter is synonymous with the terms of End Stage Renal Failure (ESRF) and End Stage Renal Disease (ESRD) which are in more widespread international usage. Within the UK, patient groups have disliked the term 'End Stage' which formerly reflected the inevitable outcome of this disease.

\section{Methods}

These analyses relate to the prevalent RRT cohort in the UK in 2007 (chapter 15 and appendix B). The cohort was defined as all adult patients prevalent on RRT on the UK Registry database on $31 / 12 / 2007$. Population estimates were obtained from the UK Office of National Statistics (ONS) [1].

Total numbers of prevalent RRT patients were calculated for the UK as a whole and by UK countries using UK Renal Registry (UKRR) data where possible but also including summary data from the centre not currently submitting data electronically. This was analysed with ONS data to calculate the prevalence of RRT pmp with 95\% confidence intervals. The numbers of prevalent patients split by dialysis modality was calculated for each centre and compared to previous years both for all centres (including percentage change from 2006 to 2007) and centres continuously reporting to the Registry since 2000 (including percentage change from 2000 to 2007). To explore the effect of centre size on modality distribution, centres were also divided into quartiles by total number of RRT patients and the proportion of patients for each modality was calculated for each quartile.

The prevalence of RRT by PCT and standardised prevalence ratios (SPR) were calculated (2008 Report appendix D www. renalreg.org). Age and gender specific prevalence was first calculated using the available Registry data on the number of prevalent patients for the covered area in England, Wales, Scotland and Northern Ireland. The data on the age and gender breakdown of the population of each PCT area was obtained from the ONS mid 2006 estimates which were derived from the 2001 census data. The age and gender specific prevalence was then used to calculate the expected prevalence for each PCT area. The age and gender standardised ratio is therefore equal to (observed prevalence)/(expected prevalence). A ratio of 1 indicates that the PCT area's prevalence was as expected if the age/gender rates found in the total covered population applied to the PCT area's population structure; a level above 1 indicates that the observed prevalence was greater than expected given the PCT area's population structure; if the lower confidence limit was above one this is statistically significant at the $5 \%$ level. The converse applies to standardised prevalence rate ratios under one. Prevalence estimates of RRT in relatively small populations such as those covered by individual Primary Care Trusts incur wide confidence intervals for any observed frequency.

To enable assessment of whether a centre was an outlier, funnel plots for smaller and larger populations have been included which show the $95 \%$ confidence intervals around the national average prevalence. PCTs in each region were then classified as having a low (below 95\% CI), normal or high (above 95\% CI) SPR.

ONS data were used to calculate the mean proportion of nonWhite people in each region weighted by PCT size. Ethnicity data were also obtained from the ONS (2001 census).

A series of analyses were performed to explore case mix differences between prevalent RRT patients. These included RRT vintage, age, gender, ethnicity, primary renal diagnosis and diabetic status (2008 Report appendix G). Patients were excluded from these analyses if the treatment modality was not known. RRT vintage was defined as median time on treatment and was calculated from the most recent start date. Vintage was calculated for each modality and the whole RRT cohort. Patients were excluded from this analysis if an accurate start date was unknown e.g. patients transferring centres. The distribution of RRT patients was analysed by age, gender, ethnicity, primary renal disease and diabetes and where appropriate split by dialysis modality. Centre level differences in age and ethnicity were also calculated.

The distribution of prevalent patients by RRT treatment modality was analysed both by centre and country. A longitudinal analysis was performed to analyse changes in use of modality for prevalent patients over time.

The data were analysed using SAS 9.1.3. A number of statistical tests were used to test for significant differences between groups. Parametric data were analysed using t-tests and Pearson correlation coefficients. Non-parametric data were analysed using Wilcoxon rank sum test and Spearman correlation coefficients.

\section{Results}

Prevalent patients numbers and changes in prevalence

The numbers of patients calculated for each country (table 4.1) (by adding the patient numbers in each renal centre) differ marginally from those quoted elsewhere when patients are allocated to areas by their individual post codes, as some centres treat patients across national boundaries.

\section{Prevalent patient numbers}

The analysis includes summary statistics from the one centre not contributing data to the UKRR, and excludes those without a treatment modality code. There were 45,484 adult patients receiving RRT in the UK at the end of 2007, giving a UK population prevalence for adults of $746 \mathrm{pmp}$ (table 4.1), an increase from $724 \mathrm{pmp}$ in 2006 [2]. Prevalence increased in each of the four UK countries and remained lower in England (736 pmp) than in Wales (798 pmp), Scotland (797 pmp) 
Table 4.1. Prevalence of RRT therapy in adults in the UK $31 / 12 / 07$

\begin{tabular}{lcccc}
\hline & England & Wales & Scotland & N Ireland \\
\hline All UK centres & 37,614 & 2,377 & 4,101 & 1,392 \\
Total population, mid-2007(millions) & 51.1 & 3.0 & 5.1 & 45,484 \\
Prevalence pmp HD & 318 & 339 & 346 & 61.0 \\
Prevalence pmp PD & 74 & 109 & 393 & 323 \\
Prevalence pmp dialysis & 392 & 448 & 423 & 76 \\
Prevalence pmp transplant & 344 & 350 & 374 & 353 \\
Prevalence pmp total & 736 & 798 & 797 & 793 \\
Confidence intervals total & $729-744$ & $766-830$ & $773-822$ & $750-833$ \\
\hline
\end{tabular}

* estimates from ONS web site

and Northern Ireland (791 pmp). Figure 4.1 shows the distribution of treatment modalities in relation to the number of prevalent RRT patients. The prevalence rate for each of the UK countries is shown in figure 4.2.

\section{Prevalent patients by RRT centre}

Both the number of prevalent patients in each renal centre and the distribution of their treatment modalities varied widely (table 4.2). Many factors contributed to this including geography, local population density, age distribution, ethnic composition and social deprivation index of that population. Local facilities, preferences and centre transplanting status also played a role in determining the modality distribution. The 23 transplant centres had higher median prevalent numbers in all modalities than non-transplanting centres $(p<0.001$

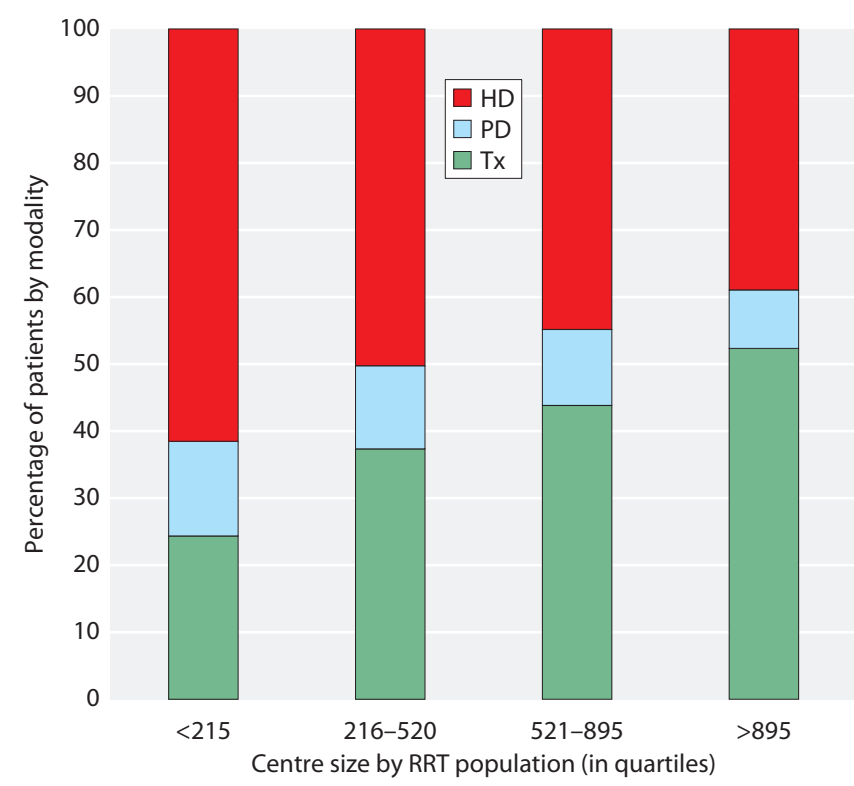

Fig. 4.1. Distribution of treatment modalities in relation to the number of prevalent RRT patients (displayed in quartiles) for all modalities), and also had a higher transplant number/dialysis number ratio (1.26 vs. 0.53 : $\mathrm{p}<0.001$ ). The wide variability in this ratio both in transplanting (0.78-2.03) and non-transplanting (0-1.08) centres suggests considerable variation in transplant follow-up policies.

The distribution of treatment modalities was also dependent on centre size, in terms of the number of RRT patients. As centre size increased the proportion of transplant patients increased at the expense of the proportion of haemodialysis patients. The proportion of transplanting centres increased through the size quartiles $(\mathrm{Q} 1=0 \%, \mathrm{Q} 2=6 \%, \mathrm{Q} 3=28 \%, \mathrm{Q} 4=94 \%)$. The only transplanting centre in Q2 was Plymouth and the only non-transplanting centre in Q4 was Carshalton (which was a transplanting centre up to 2003).

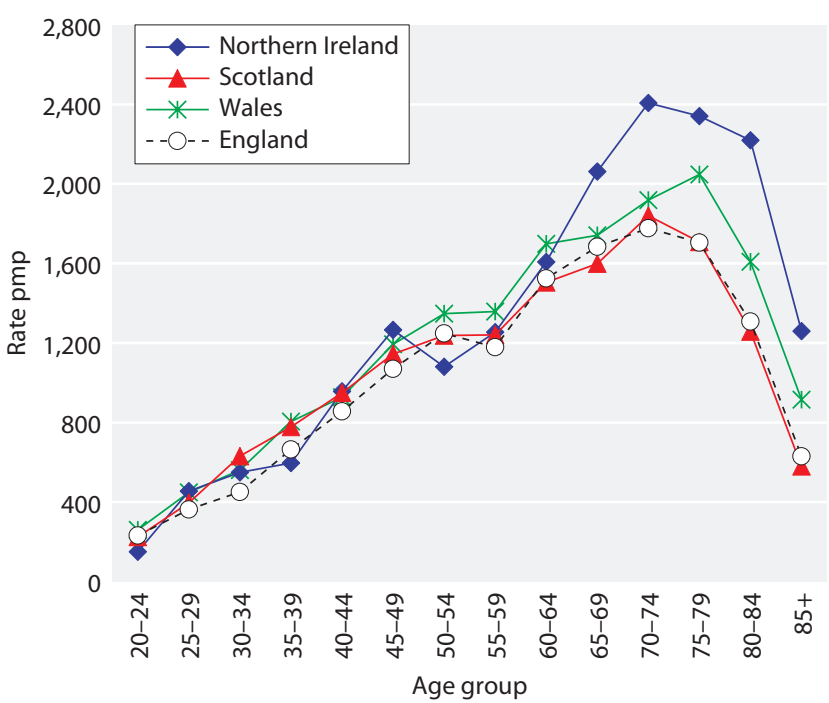

Fig. 4.2. Prevalent rate per million population by age band and UK country 
Table 4.2. Number of prevalent patients per treatment modality by centre on $31 / 12 / 07$

\begin{tabular}{|c|c|c|c|c|c|c|}
\hline Country & Centre & HD & $\mathrm{PD}$ & Dialysis & Transplant & RRT \\
\hline \multirow[t]{38}{*}{ England } & B Heart & 387 & 34 & 421 & 157 & 578 \\
\hline & $\mathrm{B} \mathrm{QEH}^{*}$ & 764 & 132 & 896 & 730 & 1,626 \\
\hline & Bradfd & 178 & 43 & 221 & 174 & 395 \\
\hline & Brightn & 333 & 87 & 420 & 265 & 685 \\
\hline & Bristol $^{*}$ & 463 & 81 & 544 & 690 & 1,234 \\
\hline & Carsh & 561 & 128 & 689 & 476 & 1,165 \\
\hline & Chelms & 108 & 42 & 150 & 38 & 188 \\
\hline & Colchester & 100 & 0 & 100 & 0 & 100 \\
\hline & Covnt* & 308 & 77 & 385 & 332 & 717 \\
\hline & Derby & 204 & 78 & 282 & 19 & 301 \\
\hline & Donc & 58 & 38 & 96 & 11 & 107 \\
\hline & Hull & 310 & 90 & 400 & 272 & 672 \\
\hline & Ipswi & 101 & 50 & 151 & 132 & 283 \\
\hline & Kent & 289 & 98 & 387 & 240 & 627 \\
\hline & L Barts* & 583 & 240 & 823 & 650 & 1,473 \\
\hline & L Guys* & 481 & 64 & 545 & 850 & 1,395 \\
\hline & L Kings & 344 & 86 & 430 & 282 & 712 \\
\hline & L RFree* & 610 & 125 & 735 & 702 & 1,437 \\
\hline & L St.G ${ }^{*}$ & 204 & 53 & 257 & 310 & 567 \\
\hline & L West* & 1,056 & 67 & 1,123 & 1,039 & 2,162 \\
\hline & Leeds* & 506 & 105 & 611 & 768 & 1,379 \\
\hline & Leic* & 675 & 203 & 878 & 716 & 1,594 \\
\hline & Liv Ain & 115 & 0 & 115 & 0 & 115 \\
\hline & Oxford ${ }^{*}$ & 342 & 147 & 489 & 839 & 1,328 \\
\hline & Plymth* & 131 & 44 & 175 & 246 & 421 \\
\hline & Ports* & 403 & 102 & 505 & 677 & 1,182 \\
\hline & Prestn & 418 & 82 & 500 & 355 & 855 \\
\hline & Redng & 230 & 98 & 328 & 217 & 545 \\
\hline & Sheff* & 566 & 93 & 659 & 513 & 1,172 \\
\hline & Shrew & 162 & 41 & 203 & 82 & 285 \\
\hline & Stevng & 329 & 43 & 372 & 176 & 548 \\
\hline & Sthend & 122 & 20 & 142 & 53 & 195 \\
\hline & Stoke & 256 & 96 & 352 & 236 & 588 \\
\hline & Sund & 165 & 15 & 180 & 164 & 344 \\
\hline & Truro & 156 & 27 & 183 & 103 & 286 \\
\hline & Wirral & 182 & 34 & 216 & 0 & 216 \\
\hline & Wolve & 275 & 62 & 337 & 104 & 441 \\
\hline & York & 115 & 26 & 141 & 90 & 231 \\
\hline \multirow[t]{5}{*}{ Wales } & Bangor & 65 & 33 & 98 & 0 & 98 \\
\hline & Cardff* & 494 & 159 & 653 & 785 & 1,438 \\
\hline & Clwyd & 71 & 19 & 90 & 65 & 155 \\
\hline & Swanse & 301 & 82 & 383 & 161 & 544 \\
\hline & Wrexm & 79 & 33 & 112 & 30 & 142 \\
\hline
\end{tabular}


Table 4.2. Continued

\begin{tabular}{|c|c|c|c|c|c|c|}
\hline Country & Centre & HD & $\mathrm{PD}$ & Dialysis & Transplant & RRT \\
\hline \multirow[t]{9}{*}{ Scotland } & Abrdn & 212 & 35 & 247 & 205 & 452 \\
\hline & Airdrie & 148 & 23 & 171 & 59 & 230 \\
\hline & D \& Gall & 50 & 16 & 66 & 11 & 77 \\
\hline & Dundee & 170 & 29 & 199 & 177 & 376 \\
\hline & Dunfn & 112 & 25 & 137 & 83 & 220 \\
\hline & Edinb* & 272 & 77 & 349 & 371 & 720 \\
\hline & Glasgw* & 599 & 104 & 703 & 902 & 1,605 \\
\hline & Inverns & 85 & 40 & 125 & 82 & 207 \\
\hline & Klmarnk & 130 & 47 & 177 & 37 & 214 \\
\hline \multirow[t]{6}{*}{ Northern Ireland } & Antrim & 129 & 16 & 145 & 55 & 200 \\
\hline & Belfast* & 262 & 63 & 325 & 423 & 748 \\
\hline & Derry & 52 & 4 & 56 & 6 & 62 \\
\hline & Newry & 86 & 14 & 100 & 47 & 147 \\
\hline & Tyrone & 83 & 5 & 88 & 61 & 149 \\
\hline & Ulster & 79 & 3 & 82 & 4 & 86 \\
\hline \multirow[t]{5}{*}{ Totals } & England & 16,227 & 3,819 & 20,046 & 17,568 & 37,614 \\
\hline & N Ireland & 691 & 105 & 796 & 596 & 1,392 \\
\hline & Scotland & 1,778 & 396 & 2,174 & 1,927 & 4,101 \\
\hline & Wales & 1,010 & 326 & 1,336 & 1,041 & 2,377 \\
\hline & UK & 19,706 & 4,646 & 24,352 & 21,132 & 45,484 \\
\hline
\end{tabular}

* Transplanting centres

Italics, centre returned summary data

Changes in prevalence

Overall growth in the prevalent UK RRT population between 2006 and 2007 was 11.8\% (table 4.3). The growth in England (13.1\%) and Wales (10.8\%) outstripped that in Scotland (5.0\%) and Northern Ireland $(2.9 \%)$. There were large variations between centres. Growth increased by $96.2 \%$ in Clwyd, $82.4 \%$ in Derry and $30.6 \%$ in London West and decreased by

Table 4.3. Number of patients on RRT by centre 2004-2007

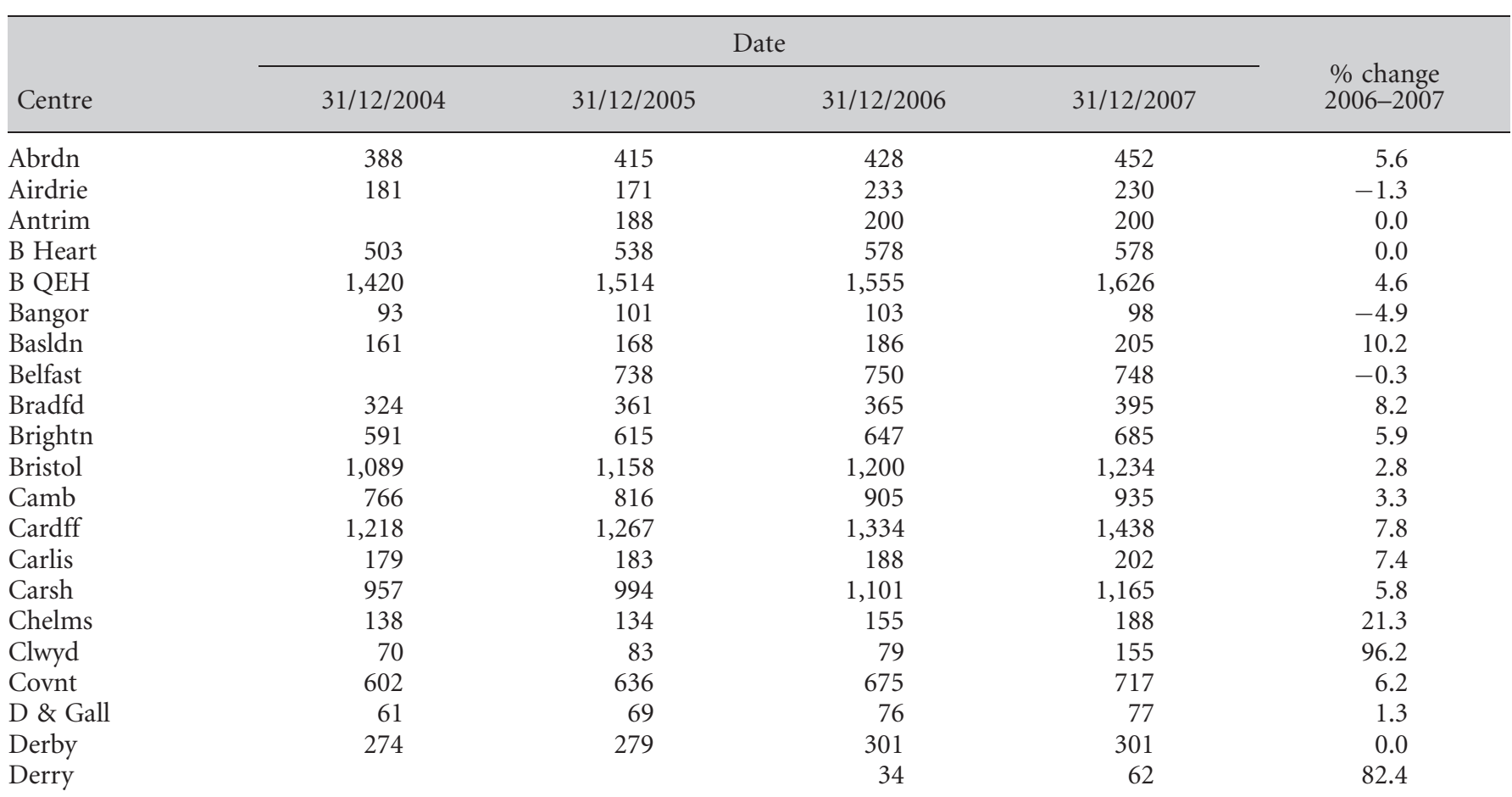


Table 4.3. Continued

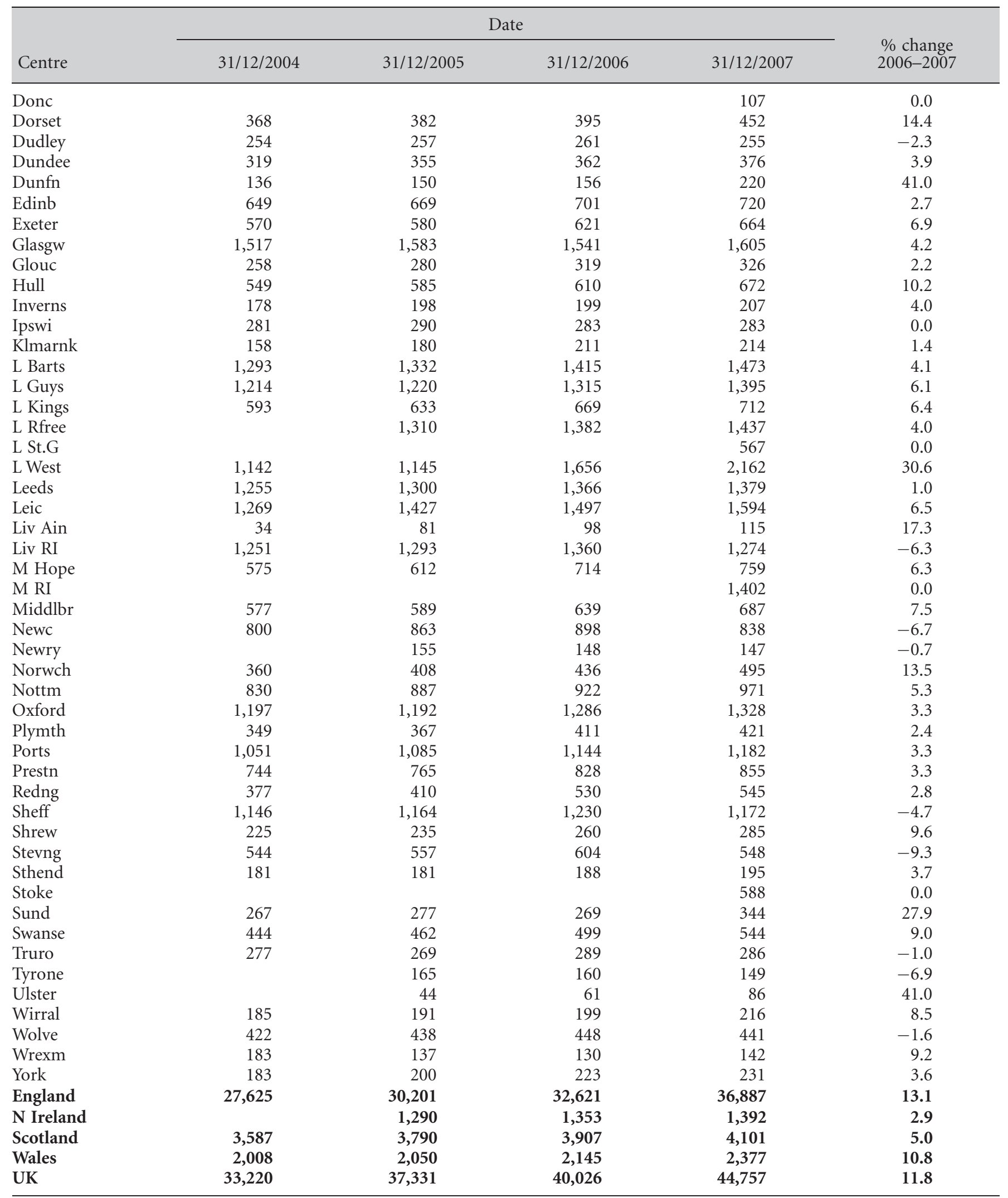


Table 4.4. Prevalent patient numbers in renal centres reporting continuously 2000-2007

\begin{tabular}{|c|c|c|c|c|c|c|c|c|c|}
\hline Centre & 2000 & 2001 & 2002 & 2003 & 2004 & 2005 & 2006 & 2007 & $\begin{array}{l}\% \text { change } \\
2000-2007\end{array}$ \\
\hline Abrdn & 302 & 316 & 355 & 349 & 388 & 415 & 428 & 452 & 49.7 \\
\hline B Heart & 422 & 452 & 444 & 497 & 503 & 538 & 578 & 578 & 37.0 \\
\hline Bristol & 905 & 945 & 991 & 1,050 & 1,089 & 1,158 & 1,200 & 1,234 & 36.4 \\
\hline Cardff & 1,028 & 1,055 & 1,092 & 1,156 & 1,218 & 1,267 & 1,334 & 1,438 & 39.9 \\
\hline Covnt & 514 & 545 & 563 & 575 & 602 & 636 & 675 & 717 & 39.5 \\
\hline D \& Gall & 54 & 72 & 73 & 79 & 61 & 69 & 76 & 77 & 42.6 \\
\hline Derby & 121 & 160 & & 259 & 274 & 279 & 301 & 301 & 148.8 \\
\hline Dudley & 244 & 235 & 231 & 241 & 254 & 257 & 261 & 255 & 4.5 \\
\hline Dundee & 236 & 244 & 288 & 299 & 319 & 355 & 362 & 376 & 59.3 \\
\hline Glouc & 235 & 195 & 210 & 243 & 258 & 280 & 319 & 326 & 38.7 \\
\hline Hull & 420 & 443 & 506 & 514 & 549 & 585 & 610 & 672 & 60.0 \\
\hline Inverns & 92 & 120 & 147 & 159 & 178 & 198 & 199 & 207 & 125.0 \\
\hline Klmarnk & 136 & 143 & 157 & 168 & 158 & 180 & 211 & 214 & 57.4 \\
\hline L Guys & 1,124 & 1,145 & 1,185 & 1,183 & 1,214 & 1,220 & 1,315 & 1,395 & 24.1 \\
\hline Leeds & 1,167 & 1,162 & 1,181 & 1,203 & 1,255 & 1,300 & 1,366 & 1,379 & 18.2 \\
\hline Leic & 973 & 1,028 & 1,079 & 1,120 & 1,269 & 1,427 & 1,497 & 1,594 & 63.8 \\
\hline Middlbr & 415 & 422 & 520 & 550 & 577 & 589 & 639 & 667 & 60.7 \\
\hline Nottm & 760 & 817 & 789 & 809 & 830 & 887 & 922 & 971 & 27.8 \\
\hline Oxford & 1,241 & 1,316 & 1,359 & 1,397 & 1,197 & 1,192 & 1,286 & 1,328 & 7.0 \\
\hline Plymth & 408 & 393 & 385 & 345 & 349 & 367 & 411 & 421 & 3.2 \\
\hline Prestn & 458 & 503 & 567 & 712 & 744 & 765 & 828 & 855 & 86.7 \\
\hline York & 92 & 124 & 160 & 186 & 183 & 200 & 223 & 231 & 151.1 \\
\hline England & 12,909 & 13,463 & 14,127 & 15,139 & 15,790 & 16,464 & 17,529 & 18,138 & 40.5 \\
\hline Scotland & 2,959 & 3,138 & 3,337 & 3,459 & 3,587 & 3,790 & 3,907 & 4,043 & 36.6 \\
\hline Wales & 1,475 & 1,640 & 1,676 & 1,770 & 1,845 & 1,866 & 1,963 & 2,124 & 44.0 \\
\hline UK & 17,343 & 18,241 & 19,140 & 20,368 & 21,222 & 22,120 & 23,399 & 24,305 & 40.1 \\
\hline
\end{tabular}

9.3\% in Stevenage. In Clwyd, the major growth was in transplant numbers, due to transfer from Liverpool of a cohort of established post-transplant patients for local follow-up. This was also true for Dunfermline where patients were transferred from Glasgow. In Derry, a new growing centre, the major growth was in the haemodialysis population. The growth in London West reflected the recent amalgamation of centres and now includes data from the transplant patients previously at London St Mary's.
In the longer term, for those 37 centres contributing data to the Registry across the 8 years between 2000 and 2007, growth in the prevalent RRT population increased by $40.1 \%$ (table 4.4), giving an average annual growth rate of around 5\%. This was fairly stable across the three UK countries whose centres submitted data over that period, ranging from $4.8 \%$ in Scotland, through $5.1 \%$ in England to $5.4 \%$ in Wales. The absolute increase of RRT patients was highest in those centres in the highest quartile (Q4) in terms of RRT 
population at baseline in 2000 (median increase $\mathrm{Q} 1=115, \mathrm{Q} 2=125, \mathrm{Q} 3=203, \mathrm{Q} 4=288$ patients), whilst the growth in percentage terms was the inverse of this (median increase Q1 $=125 \%, \mathrm{Q} 2=50 \%$, $\mathrm{Q} 3=40 \%, \mathrm{Q} 4=32 \%)$.

The long-term (1982-2007) UK prevalence pattern in relation to RRT modality is shown in figure 4.3 . The steady growth in transplant numbers was maintained but haemodialysis numbers continued to increase more rapidly. The slow contraction in home-based therapies, evident over the past decade, persisted.

Prevalence of RRT in Primary Care Trusts (PCT) in England and Health Authorities (HA) in N Ireland,

Scotland and Wales

For the first time in 2007, prevalence rates were reported in relation to the catchment area populations of Primary Care Trusts in England. Data by HA for the other UK countries continued to be reported. There were substantial variations in the crude PCT/HA area prevalence from $399 \mathrm{pmp}$ (Great Yarmouth and Waveney, population 210,600) to 1,487 pmp (Merthyr Tydfil, population 55,800). There were similar variations in SPR from 0.48 (Great Yarmouth and Waveney) to 2.44 (Heart of Birmingham) (table 4.5).

PCTs/HAs with small populations have wide confidence limits for SPR (figures 4.4 and 4.5), such that the interpretation of data from a single year may be difficult. The annual standardised prevalence rate was inherently more stable than the annual standardised acceptance rate (chapter 3), and there was a high degree of correlation between the SPR's obtained for 2007 and those calculated for the period 2002 to 2007 $\left(\mathrm{r}^{2}=0.889: \mathrm{p}<0.001\right)$.

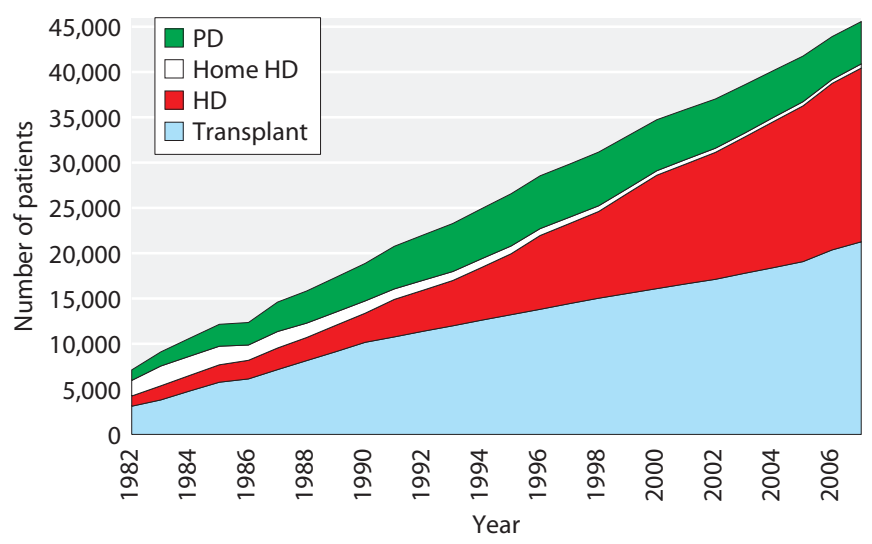

Fig. 4.3. Growth in prevalent patients, by treatment modality at the end of each year 1982-2007

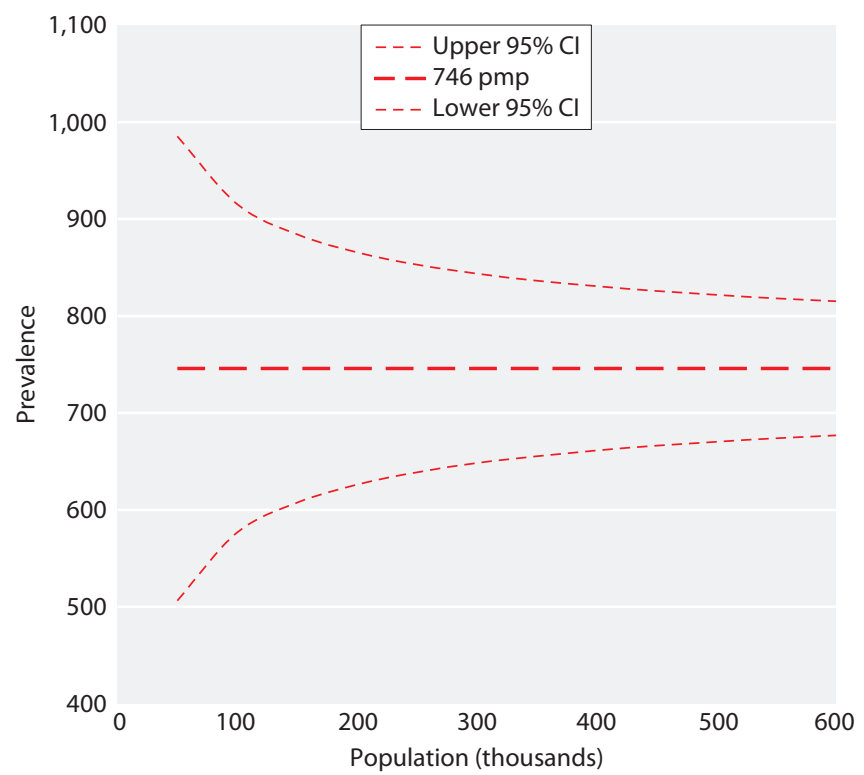

Fig. 4.4. $95 \%$ confidence limits for prevalence of $746 \mathrm{pmp}$ for population sizes $50,000-600,000$

Factors associated with variation in standardised prevalence ratios in PCTs in England and HAs in Northern Ireland, Scotland and Wales

Geographical considerations and ethnicity were the major factors underlying the variation in SPR (table 4.5). In 2007, for the PCTs/HAs with available data, there were $48 \mathrm{PCTs} / \mathrm{HAs}$ with a significantly low SPR, 129 with a normal SPR and 51 with a significantly high

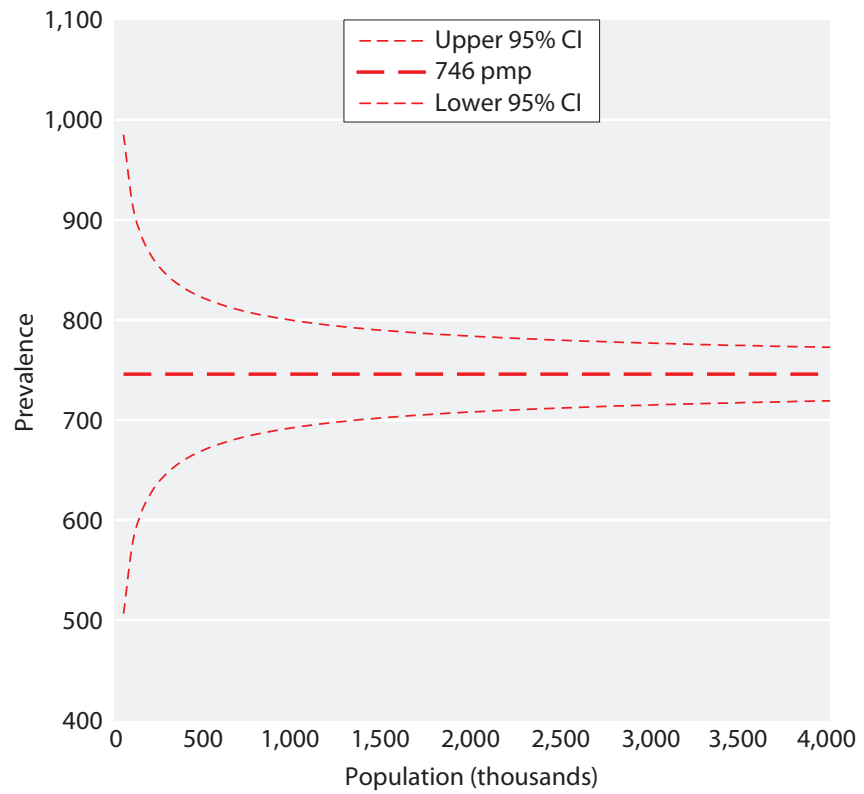

Fig. 4.5. $95 \%$ confidence limits for prevalence of $746 \mathrm{pmp}$ for population sizes $50,000-4$ million 
Table 4.5. Prevalence of RRT and standardised prevalence ratios in Primary Care Trusts and Health Authorities with complete coverage

${ }^{a}$ per million population

$\mathrm{O} / \mathrm{E}=$ standardised prevalence ratio

Blank cells - no data returned to the Registry for that year

Areas with significantly high prevalence ratios are bold in darker grey cells, areas with significantly low prevalence ratios are italicised in lighter grey cells

$\%$ non-White $=$ the sum of $\%$ South Asian and Black from the 2001 UK census

\begin{tabular}{|c|c|c|c|c|c|c|c|c|c|c|c|c|c|}
\hline \multirow[b]{2}{*}{ Region } & \multirow[b]{2}{*}{ PCT } & \multirow[b]{2}{*}{ Tot pop } & \multirow{2}{*}{$\begin{array}{l}2002 \\
\mathrm{O} / \mathrm{E}\end{array}$} & \multirow{2}{*}{$\begin{array}{l}2003 \\
\mathrm{O} / \mathrm{E}\end{array}$} & \multirow{2}{*}{$\begin{array}{l}2004 \\
\mathrm{O} / \mathrm{E}\end{array}$} & \multirow{2}{*}{$\begin{array}{r}2005 \\
\mathrm{O} / \mathrm{E}\end{array}$} & \multirow{2}{*}{$\begin{array}{l}2006 \\
\mathrm{O} / \mathrm{E}\end{array}$} & \multicolumn{4}{|c|}{2007} & \multicolumn{2}{|c|}{ All \% non } \\
\hline & & & & & & & & $\mathrm{O} / \mathrm{E}$ & LCL & UCL & $\mathrm{pmp}^{\mathrm{a}}$ & $\mathrm{O} / \mathrm{E}$ & White \\
\hline \multirow{12}{*}{$\begin{array}{l}\mathrm{NE} \\
\text { England }\end{array}$} & County Durham & 500,400 & 1.02 & 0.95 & 0.97 & 0.99 & 0.95 & 0.91 & 0.82 & 1.01 & 715 & 0.96 & 1.0 \\
\hline & Darlington & 99,100 & 0.95 & 0.97 & 0.98 & 0.96 & 0.83 & 0.83 & 0.64 & 1.06 & 636 & 0.91 & 2.1 \\
\hline & Redcar and Cleveland & 139,200 & 1.07 & 1.03 & 1.03 & 0.97 & 0.98 & 0.98 & 0.81 & 1.19 & 776 & 1.01 & 1.1 \\
\hline & Hartlepool & 91,100 & 0.94 & 0.97 & 1.02 & 0.93 & 1.00 & 0.89 & 0.69 & 1.14 & 670 & 0.96 & 1.1 \\
\hline & Middlesbrough & 138,500 & 1.21 & 1.22 & 1.09 & 1.03 & 1.06 & 1.07 & 0.88 & 1.29 & 758 & 1.11 & 6.3 \\
\hline & North Tees & 189,200 & 0.79 & 0.81 & 0.88 & 0.88 & 0.92 & 0.86 & 0.72 & 1.02 & 634 & 0.86 & 2.7 \\
\hline & Gateshead & 190,500 & 1.15 & 1.07 & 1.04 & 1.00 & 0.95 & 0.90 & 0.76 & 1.06 & 698 & 1.01 & 1.6 \\
\hline & Newcastle & 270,400 & 1.01 & 0.93 & 0.88 & 0.92 & 0.90 & 0.93 & 0.80 & 1.08 & 655 & 0.93 & 6.9 \\
\hline & North Tyneside & 195,100 & 1.05 & 1.02 & 1.00 & 1.02 & 0.99 & 0.94 & 0.80 & 1.10 & 733 & 1.00 & 1.9 \\
\hline & Northumberland & 309,900 & 0.96 & 0.93 & 0.93 & 0.89 & 0.83 & 0.81 & 0.71 & 0.93 & 671 & 0.89 & 1.0 \\
\hline & South Tyneside & 151,000 & 0.88 & 0.90 & 0.92 & 0.95 & 0.96 & 0.94 & 0.78 & 1.13 & 728 & 0.93 & 2.7 \\
\hline & Sunderland Teaching & 280,600 & 1.01 & 1.03 & 1.03 & 0.97 & 0.90 & 0.89 & 0.77 & 1.03 & 677 & 0.97 & 1.9 \\
\hline \multirow{24}{*}{$\begin{array}{l}\text { NW } \\
\text { England }\end{array}$} & Wirral & 311,100 & 1.09 & 1.09 & 1.07 & 1.04 & 1.00 & 0.93 & 0.82 & 1.06 & 726 & 1.03 & 1.7 \\
\hline & Liverpool & 436,200 & 1.21 & 1.18 & 1.19 & 1.13 & 1.14 & 1.08 & 0.97 & 1.20 & 766 & 1.15 & 5.7 \\
\hline & Central and Eastern Cheshire & 451,200 & & & & & & 0.78 & 0.69 & 0.88 & 618 & 0.78 & 1.6 \\
\hline & Western Cheshire & 235,100 & 0.99 & 0.96 & 1.01 & 0.94 & 0.91 & 0.91 & 0.78 & 1.06 & 723 & 0.95 & 1.6 \\
\hline & Knowsley & 151,500 & 1.20 & 1.24 & 1.23 & 1.15 & 1.08 & 1.04 & 0.87 & 1.26 & 752 & 1.15 & 1.6 \\
\hline & Sefton & 277,500 & 0.93 & 0.92 & 0.86 & 0.88 & 0.87 & 0.83 & 0.72 & 0.96 & 663 & 0.88 & 1.6 \\
\hline & Halton and St Helens & 297,000 & 0.87 & 0.89 & 0.87 & 0.89 & 0.95 & 0.98 & 0.85 & 1.11 & 734 & 0.91 & 1.2 \\
\hline & Warrington & 194,300 & 0.81 & 0.90 & 0.90 & 0.83 & 0.81 & 0.86 & 0.73 & 1.03 & 654 & 0.85 & 2.1 \\
\hline & Blackburn with Darwen & 141,200 & 0.86 & 1.07 & 1.14 & 1.15 & 1.20 & 1.38 & 1.16 & 1.64 & 914 & 1.15 & 22.0 \\
\hline & Blackpool & 142,800 & 0.66 & 0.76 & 0.73 & 0.70 & 0.63 & 0.76 & 0.62 & 0.94 & 609 & 0.71 & 1.6 \\
\hline & North Lancashire & 329,000 & 0.63 & 0.81 & 0.77 & 0.68 & 0.67 & 0.72 & 0.62 & 0.83 & 581 & 0.71 & 1.7 \\
\hline & Cumbria & 496,000 & 0.77 & 0.81 & 0.78 & 0.76 & 0.76 & 0.75 & 0.67 & 0.84 & 619 & 0.77 & 0.7 \\
\hline & Central Lancashire & 451,600 & 0.65 & 0.71 & 0.74 & 0.74 & 0.72 & 0.78 & 0.69 & 0.88 & 591 & 0.73 & 5.6 \\
\hline & East Lancashire & 384,500 & 0.67 & 0.88 & 0.94 & 0.89 & 0.93 & 1.05 & 0.94 & 1.18 & 783 & 0.90 & 8.1 \\
\hline & Ashton, Leigh and Wigan & 305,500 & & 0.61 & 0.64 & 0.66 & 0.69 & 0.91 & 0.80 & 1.05 & 694 & 0.71 & 1.3 \\
\hline & Bolton & 262,500 & & 0.75 & 0.75 & 0.81 & 0.83 & 1.08 & 0.94 & 1.23 & 789 & 0.85 & 11.0 \\
\hline & Bury & 182,900 & & 0.36 & 0.42 & 0.45 & 0.45 & 0.89 & 0.74 & 1.06 & 656 & 0.53 & 6.1 \\
\hline & Manchester & 451,900 & & & & & & 1.07 & 0.95 & 1.19 & 659 & 1.07 & 19.0 \\
\hline & Heywood, Middleton and Rochdale & 206,400 & & & & & & 0.99 & 0.84 & 1.16 & 707 & 0.99 & 11.4 \\
\hline & Oldham & 219,800 & & 0.49 & 0.54 & 0.50 & 0.60 & 0.91 & 0.77 & 1.07 & 641 & 0.62 & 13.9 \\
\hline & Salford & 217,800 & & 0.71 & 0.64 & 0.62 & 0.66 & 0.78 & 0.66 & 0.94 & 565 & 0.69 & 3.9 \\
\hline & Stockport & 280,800 & & & & & & 0.84 & 0.72 & 0.97 & 648 & 0.84 & 4.3 \\
\hline & Tameside and Glossop & 247,700 & & & & & & 0.97 & 0.83 & 1.12 & 715 & 0.97 & 4.9 \\
\hline & Trafford & 212,100 & & & & & & 0.78 & 0.65 & 0.93 & 585 & 0.78 & 8.4 \\
\hline \multirow{7}{*}{$\begin{array}{l}\text { Yorkshire } \\
\text { \& Humber }\end{array}$} & East Riding of Yorkshire & 331,100 & 0.84 & 0.84 & 0.81 & 0.82 & 0.78 & 0.78 & 0.68 & 0.89 & 649 & 0.81 & 1.2 \\
\hline & Hull & 256,200 & 1.04 & 0.98 & 1.02 & 1.02 & 0.97 & 1.00 & 0.86 & 1.16 & 703 & 1.00 & 2.3 \\
\hline & North East Lincolnshire & 159,900 & 0.93 & 0.94 & 1.00 & 1.00 & 0.97 & 0.99 & 0.83 & 1.18 & 750 & 0.97 & 1.4 \\
\hline & North Lincolnshire & 155,200 & 1.01 & 0.98 & 0.96 & 0.91 & 0.94 & 0.92 & 0.76 & 1.10 & 728 & 0.95 & 2.5 \\
\hline & North Yorkshire and York & 783,200 & 0.84 & 0.84 & 0.85 & 0.83 & 0.82 & 0.82 & 0.75 & 0.89 & 650 & 0.83 & 1.4 \\
\hline & Barnsley & 223,700 & 1.17 & 1.19 & 1.21 & 1.11 & 1.08 & 1.02 & 0.88 & 1.18 & 782 & 1.12 & 0.9 \\
\hline & Doncaster & 290,400 & 1.04 & 1.12 & 1.10 & 1.01 & 1.02 & 0.93 & 0.82 & 1.07 & 716 & 1.03 & 2.3 \\
\hline
\end{tabular}


Table 4.5. Continued

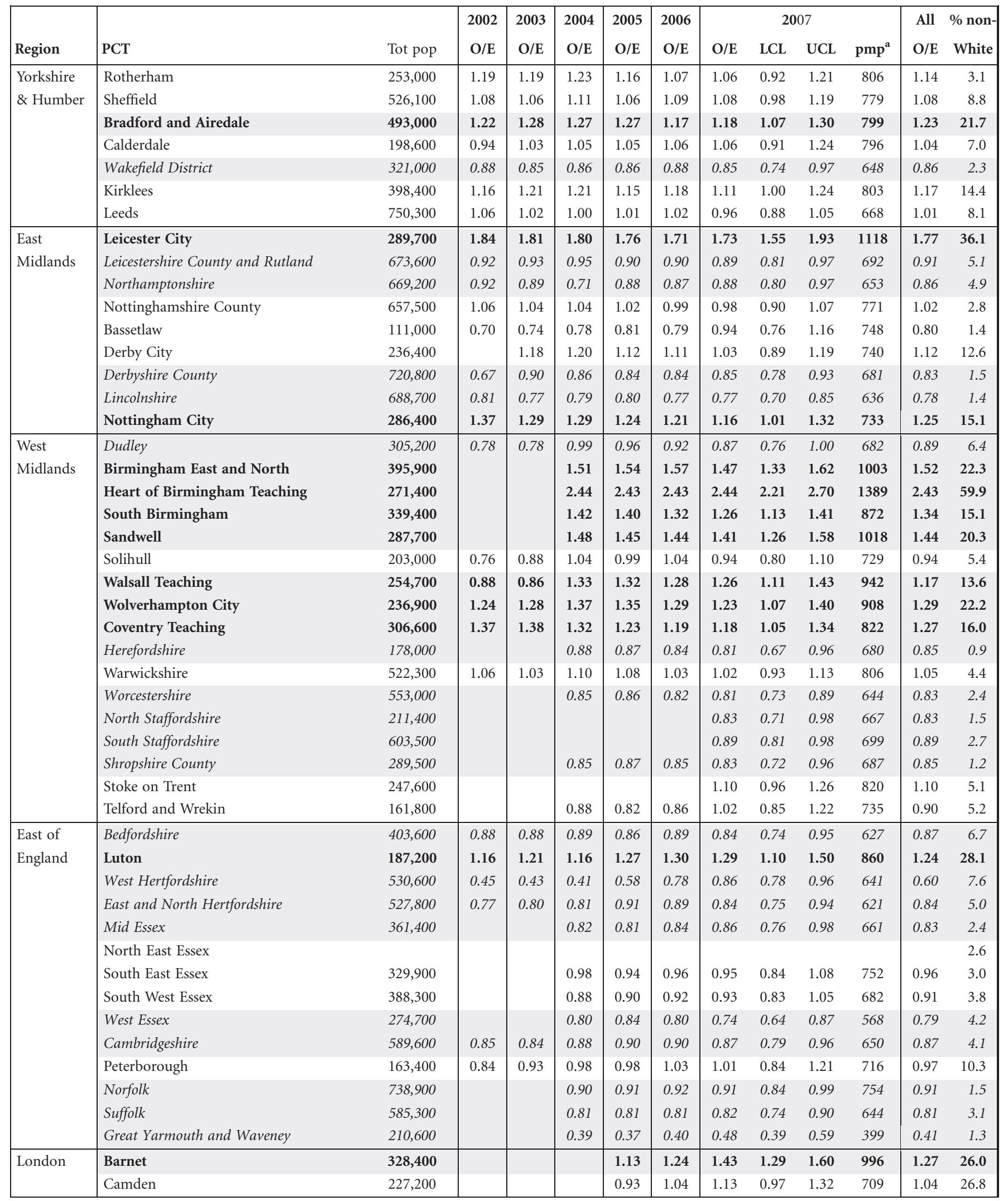


Table 4.5. Continued

\begin{tabular}{|c|c|c|c|c|c|c|c|c|c|c|c|c|c|}
\hline \multirow[b]{2}{*}{ Region } & \multirow[b]{2}{*}{ PCT } & \multirow[b]{2}{*}{ Tot pop } & \multirow{2}{*}{$\begin{array}{l}2002 \\
\mathrm{O} / \mathrm{E}\end{array}$} & \multirow{2}{*}{$\begin{array}{l}2003 \\
\mathrm{O} / \mathrm{E}\end{array}$} & \multirow{2}{*}{$\begin{array}{c}2004 \\
\mathrm{O} / \mathrm{E}\end{array}$} & \multirow{2}{*}{$\begin{array}{l}2005 \\
\mathrm{O} / \mathrm{E}\end{array}$} & \multirow{2}{*}{$\begin{array}{l}2006 \\
\mathrm{O} / \mathrm{E}\end{array}$} & \multicolumn{4}{|c|}{2007} & \multirow{2}{*}{$\begin{array}{l}\text { All } \\
\mathrm{O} / \mathrm{E}\end{array}$} & \multirow{2}{*}{$\begin{array}{l}\text { \% non } \\
\text { White }\end{array}$} \\
\hline & & & & & & & & $\mathrm{O} / \mathrm{E}$ & LCL & UCL & $\mathrm{pmp}^{\mathrm{a}}$ & & \\
\hline \multirow[t]{29}{*}{ London } & Enfield & 285,400 & & & & 1.46 & 1.46 & 1.41 & 1.25 & 1.58 & 978 & 1.44 & 22.9 \\
\hline & Haringey Teaching & 225,600 & & & & 1.50 & 1.53 & 1.54 & 1.35 & 1.76 & 971 & 1.52 & 34.4 \\
\hline & Islington & 185,500 & & & & 1.33 & 1.45 & 1.38 & 1.18 & 1.61 & 868 & 1.39 & 24.6 \\
\hline & Barking and Dagenham & 165,400 & & & 1.11 & 1.14 & 1.14 & 1.12 & 0.93 & 1.34 & 719 & 1.13 & 14.8 \\
\hline & City and Hackney Teaching & 216,200 & & & & & 1.44 & 1.44 & 1.25 & 1.66 & 874 & 1.44 & 39.7 \\
\hline & Havering & 227,500 & & & & & & 0.77 & 0.65 & 0.92 & 598 & 0.77 & 4.8 \\
\hline & Newham & 248,300 & & & 1.41 & 1.60 & 1.74 & 1.78 & 1.57 & 2.01 & 1019 & 1.64 & 60.6 \\
\hline & Redbridge & 251,800 & & & 1.16 & 1.25 & 1.22 & 1.23 & 1.07 & 1.41 & 838 & 1.22 & 36.5 \\
\hline & Tower Hamlets & 212,500 & & & 1.12 & 1.14 & 1.22 & 1.29 & 1.10 & 1.51 & 729 & 1.20 & 48.6 \\
\hline & Waltham Forest & 222,100 & & & & & 1.34 & 1.51 & 1.32 & 1.73 & 977 & 1.43 & 35.5 \\
\hline & Brent Teaching & 271,400 & & & & & 1.37 & 2.03 & 1.83 & 2.24 & 1360 & 1.71 & 54.7 \\
\hline & Ealing & 306,400 & 1.50 & 1.44 & 1.53 & 1.49 & 1.61 & 1.67 & 1.50 & 1.85 & 1119 & 1.55 & 41.3 \\
\hline & Hammersmith and Fulham & 171,400 & 1.41 & 1.45 & 1.51 & 1.37 & 1.33 & 1.25 & 1.06 & 1.47 & 805 & 1.38 & 22.2 \\
\hline & Harrow & 214,600 & & & & & & 1.71 & 1.51 & 1.93 & 1216 & 1.71 & 41.2 \\
\hline & Hillingdon & 250,100 & & & 0.89 & 0.99 & 1.07 & 0.95 & 0.82 & 1.11 & 660 & 0.98 & 20.9 \\
\hline & Hounslow & 218,600 & & & 1.68 & 1.62 & 1.51 & 1.42 & 1.24 & 1.63 & 947 & 1.55 & 35.1 \\
\hline & Kensington and Chelsea & 178,000 & & & & & & 0.75 & 0.61 & 0.92 & 528 & 0.75 & 21.4 \\
\hline & Westminster & 231,700 & & & & & & 1.00 & 0.85 & 1.17 & 673 & 1.00 & 26.8 \\
\hline & Bexley & 221,600 & 1.19 & 1.21 & 1.14 & 1.10 & 1.14 & 1.14 & 0.99 & 1.32 & 848 & 1.15 & 8.6 \\
\hline & Bromley & 299,400 & 1.00 & 1.00 & 1.00 & 0.99 & 0.99 & 0.94 & 0.82 & 1.07 & 708 & 0.98 & 8.4 \\
\hline & Greenwich Teaching & 222,600 & 1.06 & 1.03 & 0.92 & 1.12 & 1.12 & 1.17 & 1.01 & 1.36 & 764 & 1.08 & 22.9 \\
\hline & Lambeth & 272,200 & 1.33 & 1.33 & 1.36 & 1.36 & 1.37 & 1.66 & 1.48 & 1.87 & 1032 & 1.41 & 37.6 \\
\hline & Lewisham & 255,600 & 1.59 & 1.53 & 1.63 & 1.65 & 1.67 & 1.72 & 1.53 & 1.93 & 1103 & 1.64 & 34.1 \\
\hline & Southwark & 269,000 & 1.55 & 1.58 & 1.57 & 1.61 & 1.60 & 1.72 & 1.53 & 1.93 & 1078 & 1.61 & 37.0 \\
\hline & Croydon & 337,000 & 1.00 & 1.09 & 1.13 & 1.18 & 1.17 & 1.32 & 1.18 & 1.48 & 920 & 1.16 & 29.8 \\
\hline & Kingston & 156,000 & & & & & & 1.06 & 0.89 & 1.28 & 731 & 1.06 & 15.5 \\
\hline & Richmond and Twickenham & 179,500 & & & & & & 0.70 & 0.57 & 0.86 & 501 & 0.70 & 9.0 \\
\hline & Sutton and Merton & 382,000 & & & & & & 1.20 & 1.07 & 1.34 & 832 & 1.20 & 18.1 \\
\hline & Wandsworth & 279,200 & & & & & & 1.38 & 1.22 & 1.57 & 867 & 1.38 & 22.0 \\
\hline \multirow{17}{*}{$\begin{array}{l}\text { SE } \\
\text { England }\end{array}$} & Isle of Wight National Health Service & 138,200 & 0.71 & 0.75 & 0.73 & 0.63 & 0.63 & 0.58 & 0.46 & 0.74 & 499 & 0.67 & 1.3 \\
\hline & Hampshire & $1,265,900$ & 0.76 & 0.78 & 0.78 & 0.76 & 0.78 & 0.76 & 0.71 & 0.82 & 593 & 0.77 & 2.2 \\
\hline & Portsmouth City Teaching & 196,300 & 1.16 & 1.12 & 1.09 & 1.05 & 0.99 & 0.98 & 0.83 & 1.17 & 667 & 1.06 & 5.3 \\
\hline & Southampton City & 229,100 & 0.90 & 0.92 & 0.95 & 0.92 & 0.90 & 0.90 & 0.77 & 1.07 & 602 & 0.91 & 7.6 \\
\hline & West Kent & & & & & & & & & & & & 3.9 \\
\hline & Medway & & & & & & & & & & & & 5.4 \\
\hline & Eastern and Coastal Kent & & & & & & & & & & & & 2.4 \\
\hline & Hastings and Rother & 176,200 & & & 0.85 & 0.79 & 0.78 & 0.72 & 0.60 & 0.88 & 607 & 0.78 & 2.4 \\
\hline & Brighton and Hove City & 251,500 & & & 0.85 & 0.84 & 0.87 & 0.86 & 0.73 & 1.00 & 608 & 0.85 & 5.7 \\
\hline & East Sussex Downs and Weald & 330,200 & & & 0.84 & 0.81 & 0.77 & 0.80 & 0.70 & 0.91 & 666 & 0.80 & 2.3 \\
\hline & Surrey & $1,073,400$ & & & 0.76 & 0.76 & 0.78 & 0.86 & 0.80 & 0.93 & 661 & 0.79 & 4.9 \\
\hline & West Sussex & 770,600 & & & 0.77 & 0.76 & 0.76 & 0.80 & 0.73 & 0.87 & 644 & 0.77 & 3.4 \\
\hline & Milton Keynes & 230,100 & 0.90 & 0.97 & 0.97 & 0.95 & 0.90 & 0.96 & 0.81 & 1.12 & 656 & 0.94 & 9.1 \\
\hline & Berkshire East & 382,200 & 1.04 & 1.06 & 1.10 & 1.07 & 1.11 & 1.24 & 1.11 & 1.38 & 866 & 1.11 & 16.0 \\
\hline & Berkshire West & 445,400 & 0.98 & 1.01 & 1.05 & 0.99 & 1.03 & 1.13 & 1.02 & 1.25 & 806 & 1.04 & 7.3 \\
\hline & Oxfordshire & 607,400 & 1.09 & 1.12 & 1.10 & 1.04 & 1.04 & 0.96 & 0.87 & 1.05 & 693 & 1.05 & 5.0 \\
\hline & Buckinghamshire & 500,700 & 1.03 & 1.02 & 1.00 & 0.99 & 0.98 & 0.96 & 0.87 & 1.07 & 727 & 1.00 & 7.7 \\
\hline SW & Bath and North East Somerset & 175,600 & 0.68 & 0.70 & 0.82 & 0.86 & 0.84 & 0.85 & 0.71 & 1.03 & 644 & 0.80 & 2.8 \\
\hline England & Bristol & 410,700 & 1.34 & 1.36 & 1.35 & 1.30 & 1.30 & 1.22 & 1.10 & 1.36 & 825 & 1.31 & 8.2 \\
\hline
\end{tabular}


Table 4.5. Continued

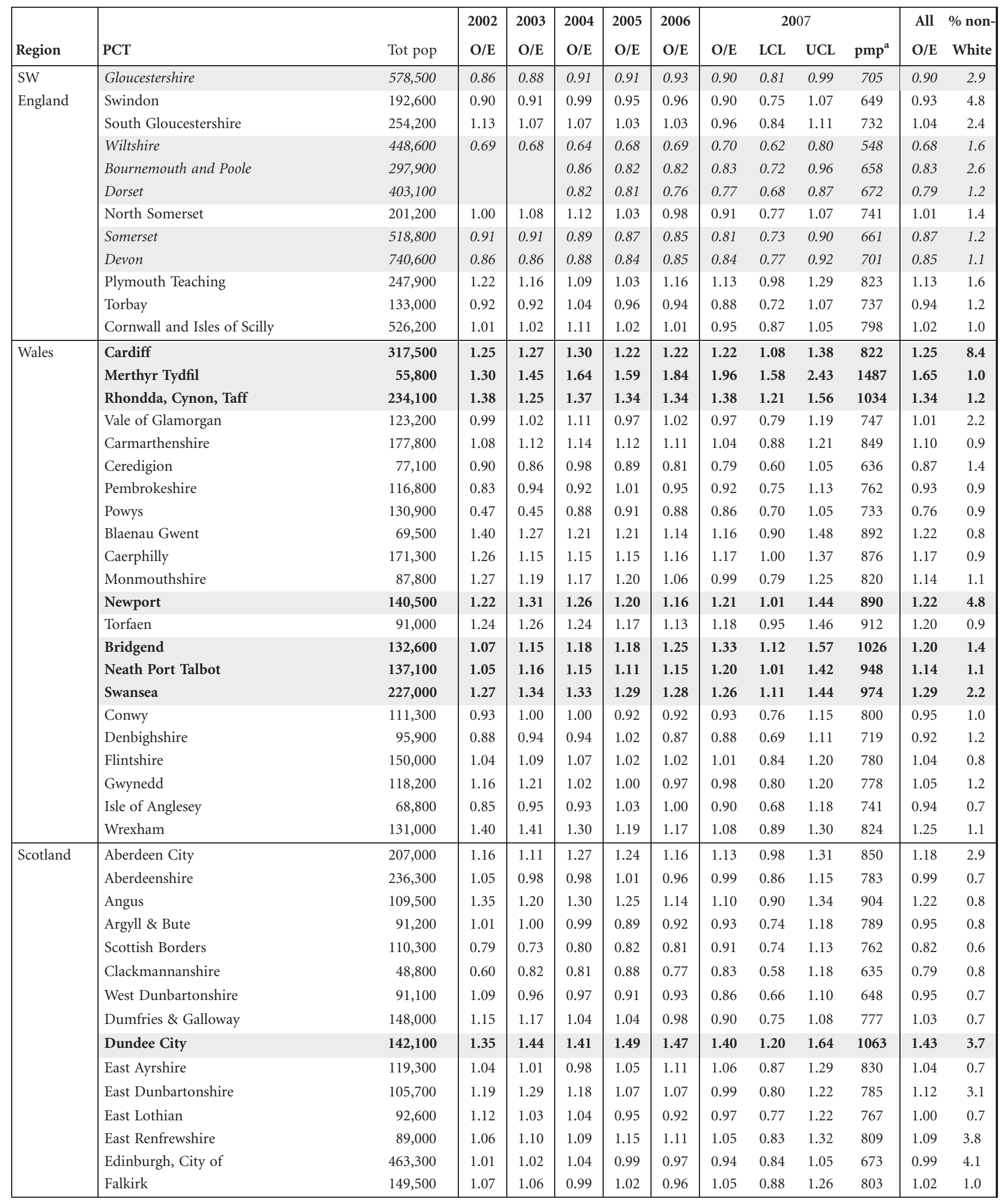


Table 4.5. Continued

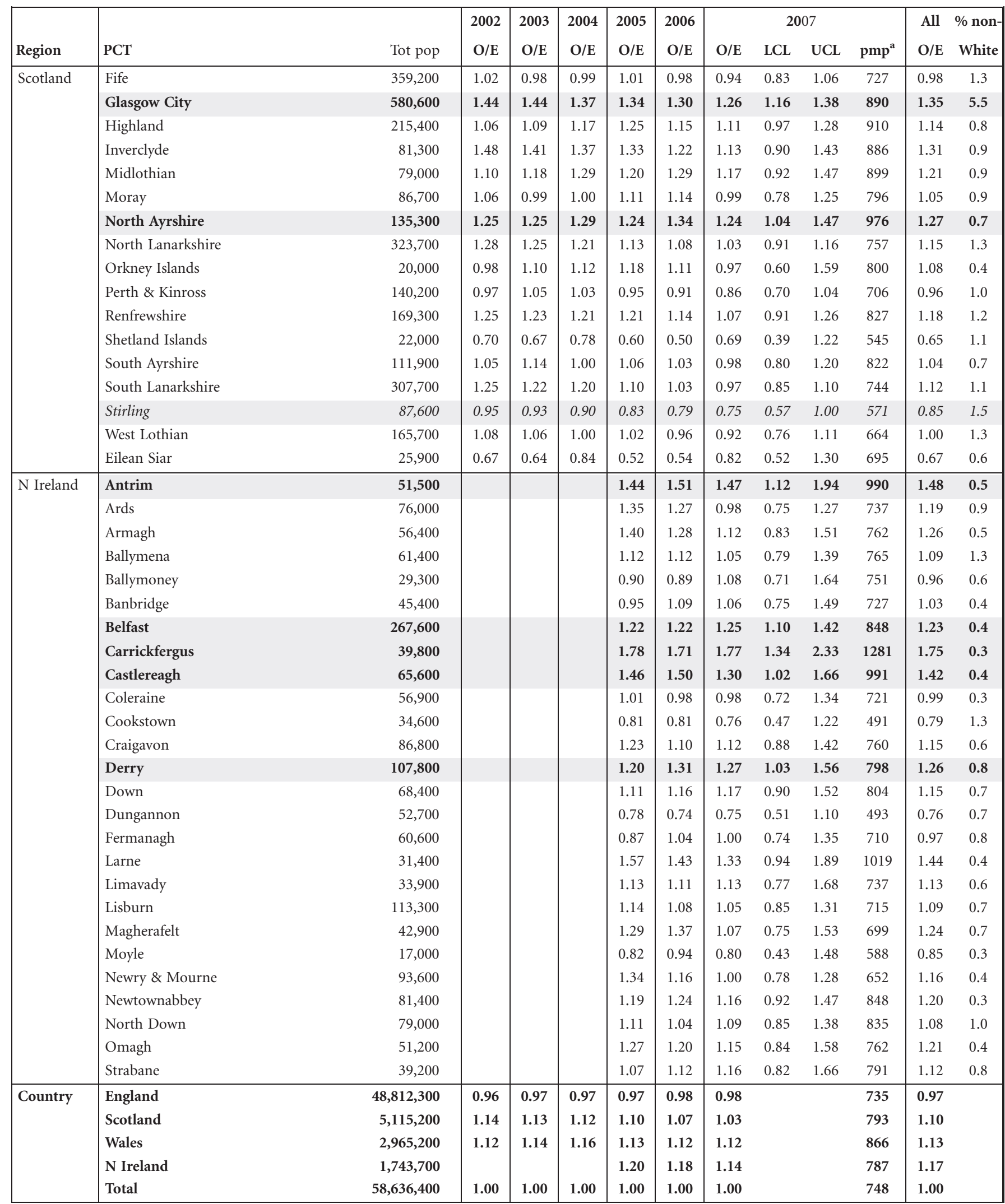


Table 4.6. Summary regional distribution of PCTs and HAs with significantly low, normal or significantly high values of SPR and mean (weighted by PCT size) \% non-Whites per region on 31/12/07

\begin{tabular}{|c|c|c|c|c|c|c|}
\hline Region & \multicolumn{3}{|c|}{ Prevalence group } & Total & $\begin{array}{c}\text { Mean \% } \\
\text { non-White }\end{array}$ & $\begin{array}{l}\text { Weighted mean } \\
\% \text { non-White }\end{array}$ \\
\hline NW England & 9 & 14 & 1 & 24 & 5.9 & 5.6 \\
\hline Yorkshire \& Humber & 3 & 10 & 1 & 14 & 5.5 & 6.5 \\
\hline East Midlands & 4 & 3 & 2 & 9 & 9.0 & 6.6 \\
\hline London & 3 & 7 & 21 & 31 & 28.5 & 28.9 \\
\hline SE England & 6 & 6 & 2 & 14 & 5.7 & 5.3 \\
\hline SW England & 6 & 7 & 1 & 14 & 2.4 & 2.3 \\
\hline England & 47 & 65 & 36 & 148 & 10.9 & 9.4 \\
\hline Wales & 0 & 15 & 7 & 22 & 1.6 & 2.1 \\
\hline Scotland & 1 & 28 & 3 & 32 & 1.4 & 2.0 \\
\hline
\end{tabular}

SPR. The geographical distribution of these is summarised in table 4.6. East of England $(\mathrm{p}<0.001)$, the South East and South West of England $(\mathrm{p}<0.08)$ had a higher proportion of areas with a low SPR compared with the UK as a whole, whilst in London $(\mathrm{p}<0.001)$ there was a significantly higher proportion of areas with a high SPR. The West Midlands (41\%) and Wales $(32 \%)$ had a relatively higher percentage of PCTs/HAs with high SPRs compared to the rest of the UK (22\%) but not significantly so. In Wales $(p<0.01)$, Scotland $(\mathrm{p}<0.001)$ and Northern Ireland $(\mathrm{p}<0.01)$ there were significantly fewer PCTs/HAs that had low values than in the rest of the UK.

PCTs/HAs with a high SPR had significantly higher ethnic minority populations than those with significantly low or normal SPRs $(\mathrm{p}<0.0001)$ (figures 4.6, 4.7a and b). Mean SPR was significantly higher in the 47 PCTs/ HAs with an ethnic minority population greater than $10 \%$ than in those with lower ethnic minority populations ( 1.33 vs. $0.97: \mathrm{p}<0.001)$. The SPR $(\mathrm{r}=0.257$, $\mathrm{p}<0.001)$ was highly correlated with ethnicity. For each $10 \%$ increase in ethnic minority population the age standardised prevalence ratio increased by 0.2 .

The relationship between the ethnic composition of a PCT/HA area and its SPR is further demonstrated in figure $4.7 \mathrm{a}$, which shows the relationship for all PCTs/ HAs and in figure $4.7 \mathrm{~b}$ where those with $<1 \%$ ethnic minority populations have been excluded.

Only 1 (Kensington \& Chelsea, an area of low social deprivation) of the $47 \mathrm{PCT} / \mathrm{HA}$ areas with ethnic minority populations greater than $10 \%$ had a low SPR, whereas 34 had high SPRs. In contrast only 17 of the $181 \mathrm{PCT} / \mathrm{HA}$ areas with ethnic minority populations less than $10 \%$ had high SPRs. Seven of these were in Wales (Cardiff, Methyr Tydfil, Rhondda-Cynon-Taff, Newport, Bridgend, Neath and Port Talbot), 3 in Scotland (Dundee City, Glasgow City, North Ayrshire) and 5 in Northern Ireland (Antrim, Belfast, Carrickfergus, Castlereagh and Derry). The only centres in England were Bristol and Berkshire West. The factors contributing to these regional disparities remained unclear but social deprivation was likely to be an important factor.

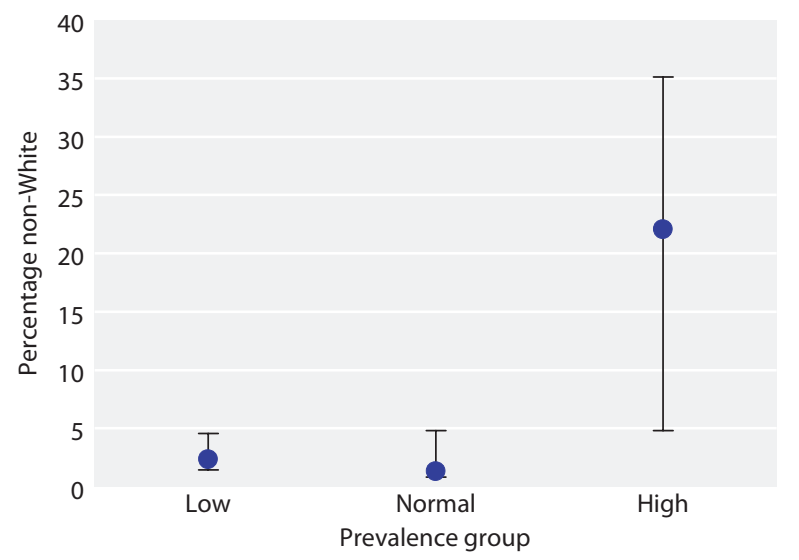

Fig. 4.6. Percentage non-Whites in PCTs and HAs with significantly low, normal and significantly high SPR values (median and quartiles) 


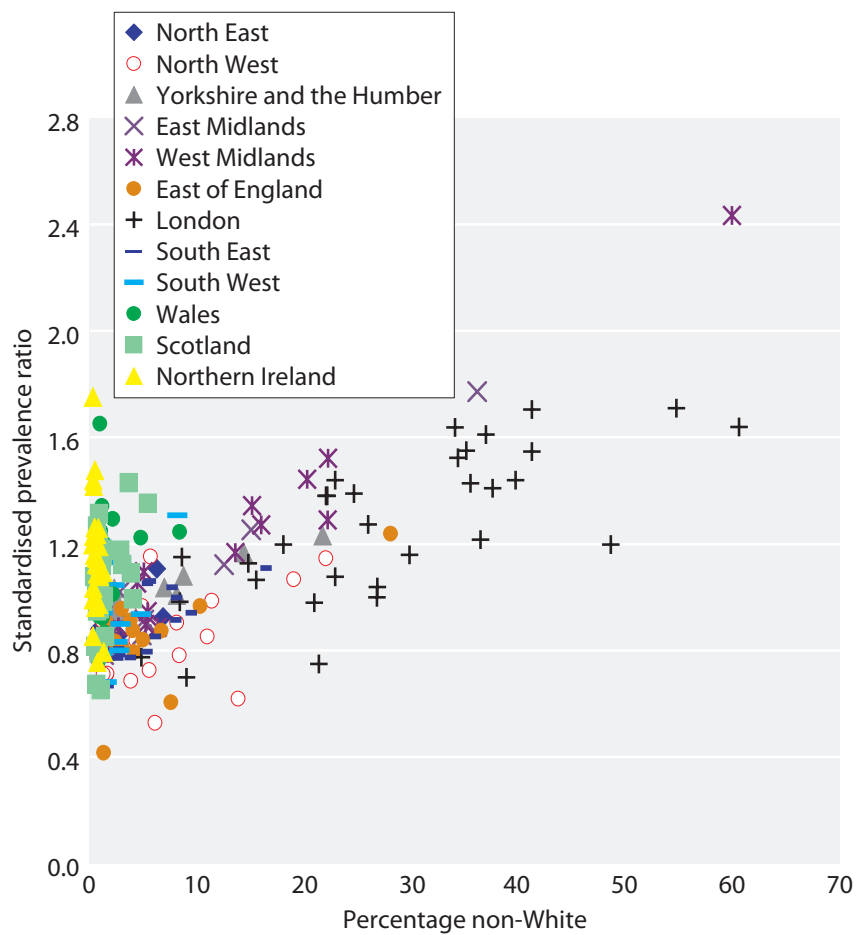

Fig. 4.7a. Ethnicity and standardised prevalence ratios for all PCTs and HAs by percentage non-White with available data

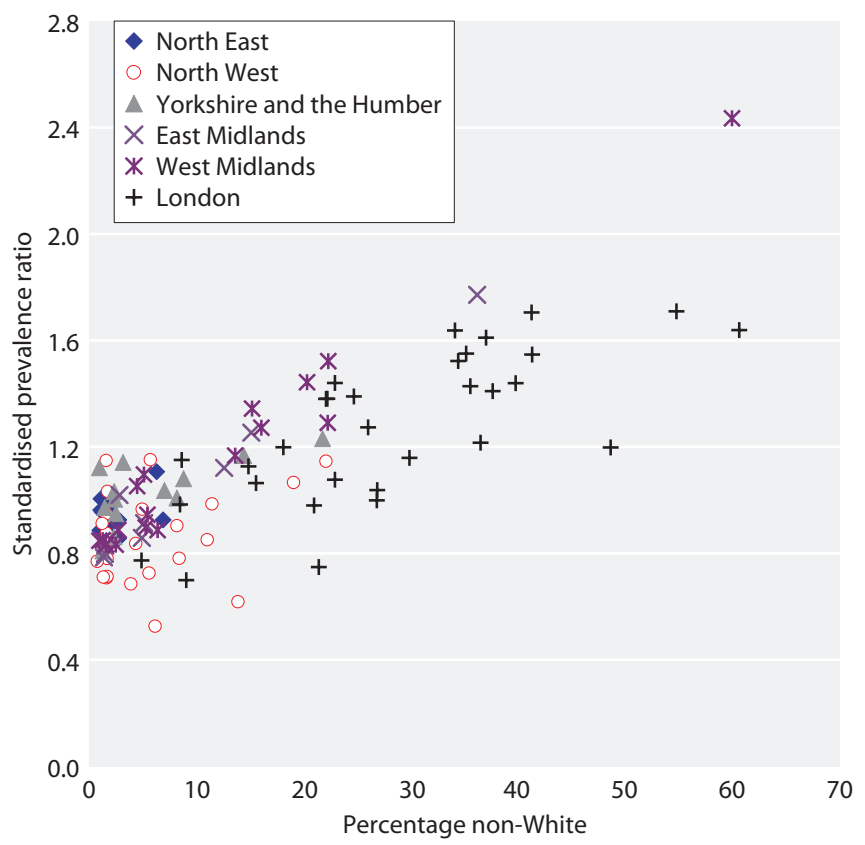

Fig. 4.7b. Ethnicity and standardised prevalence ratios for all PCTs and HAs by percentage non-White (excluding low percentage ethnic minority areas)

\section{Case mix in prevalent $R R T$ patients}

Vintage

For patients who recover for $>90$ days and then restart RRT, median time from the start of RRT was calculated from the most recent start date. table 4.7 shows the median vintage (years since starting RRT) of prevalent RRT patients in 2007. Median vintage of the whole RRT cohort was 5.3 years. Patients with functioning transplants had survived a median 10.4 years on RRT whilst the median vintage of $\mathrm{HD}$ and $\mathrm{PD}$ patients was much less (2.8 and 2.1 years respectively). There was no significant change from 2006 [2].

Age

The median age of prevalent UK patients on RRT in 2007 was unchanged compared to 2006 at 57 years (table 4.8) [2]. The age profile varied markedly with modality. The median age of patients on HD (65.2 yrs) was greater than those on PD (60.3 yrs) and substantially higher than that of transplanted patients (50.2 yrs). These were all minimally higher than those reported in 2006. HD patients in Wales and Northern Ireland and PD patients in Wales were slightly older than in the rest of the UK.

There were however wide inter-centre variations in the median age of their RRT population (51.5 to $70.8 \mathrm{yrs}$ ), the median age being less in transplanting than in nontransplanting centres $(55.5$ vs. 60.8 yrs: $\mathrm{p}<0.001)$. The median age of HD patients was slightly less in transplanting than in non-transplanting centres (62.2 vs. 64.2: $\mathrm{p}<0.05)$, but there was no difference in the median ages of PD and transplant patients. This implies that the major factor accounting for the lower median age of RRT patients in transplanting centres was the higher number of transplant patients under follow-up in transplant centres. The differing age distributions of the transplant and dialysis populations are illustrated in figure 4.8, demonstrating that patient age at peak dialysis prevalence was around three decades higher than patient age at peak transplant prevalence.

Table 4.7. Median vintage of prevalent RRT patients on $31 / 12 / 07$

\begin{tabular}{lrc}
\hline Modality & N & $\begin{array}{c}\text { Median time treated } \\
\text { (years) }\end{array}$ \\
\hline Haemodialysis & 18,825 & 2.8 \\
Peritoneal dialysis & 4,495 & 2.1 \\
Transplant & 19,443 & 10.4 \\
All RRT & $\mathbf{4 2 , 7 6 3}$ & $\mathbf{5 . 3}$ \\
\hline
\end{tabular}


Table 4.8. Median age of prevalent RRT patients by treatment modality by renal centre on 31/12/07

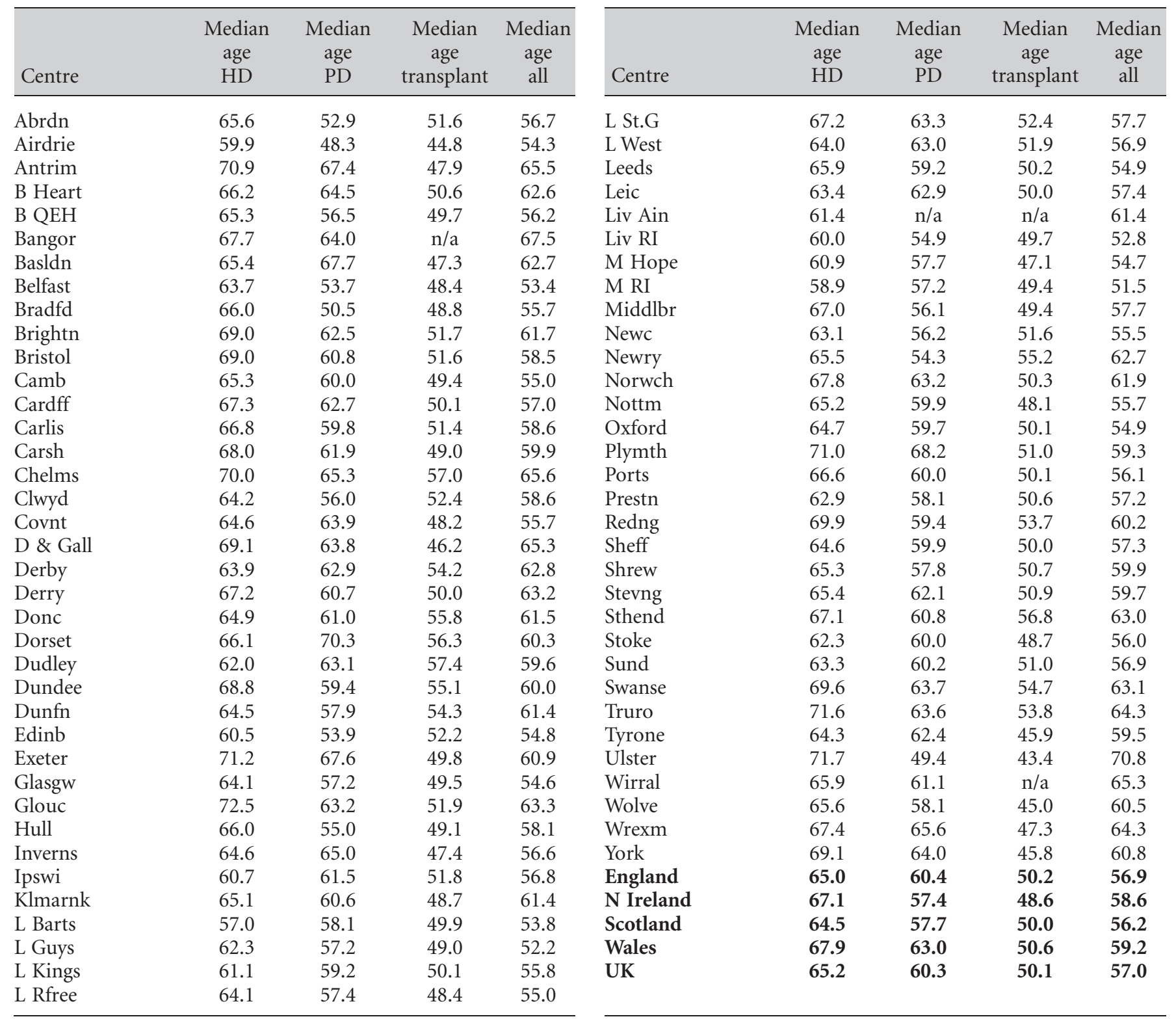

n/a not applicable

Gender

In the UK in 2007, age at peak absolute RRT prevalence was in the 55-65 year age-band in males and females (figure 4.9).

Correcting this for the age and gender distribution of the UK population calculated from PCT/ HA populations covered by the Registry using 2001 census data allowed estimation of crude prevalence rates by age and gender (figure 4.10).
The overall UK peak crude prevalence rate occurred in the age band $70-74$ at 1,808 pmp. For all ages, crude prevalence rates in males exceeded those in females, peaking in the 75-79 year age band for males at $2,506 \mathrm{pmp}$ and in females in the 70-74 year age band at $1,314 \mathrm{pmp}$.

The male:female ratio of the crude prevalence rate remained stable at around 1.5 until the 60-65 age band, then increased markedly to 1.8 in the $65-74$ age 


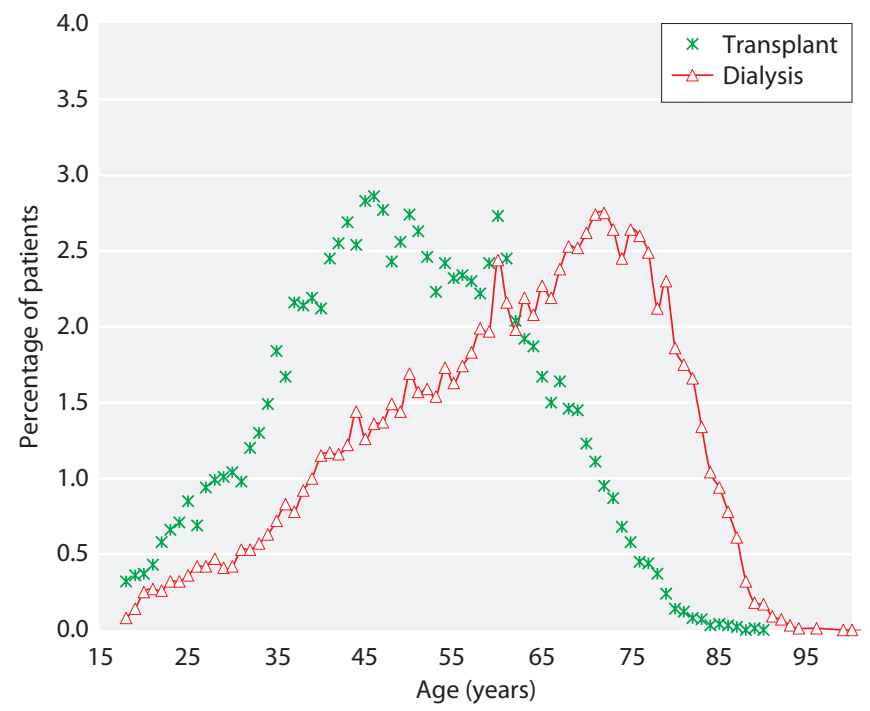

Fig. 4.8. Age profile of prevalent RRT patients on 31/12/07

band, 2.2 at $75-79$ years, 2.7 at $80-84$ years and 4.7 in those over 85 years (figure 4.11).

\section{Ethnicity}

Thirty-seven of the 71 centres submitting electronic data to the UKRR in 2007 provided ethnicity data that were at least $90 \%$ complete (table 4.9 ), slightly worse than in 2006 [2]. Data from 60 centres had greater than $50 \%$ returns. In the whole UK, $18.6 \%$ of the prevalent RRT population were from an ethnic minority, similar to the proportion in England. The proportions in Wales, Scotland and Northern Ireland were very small,

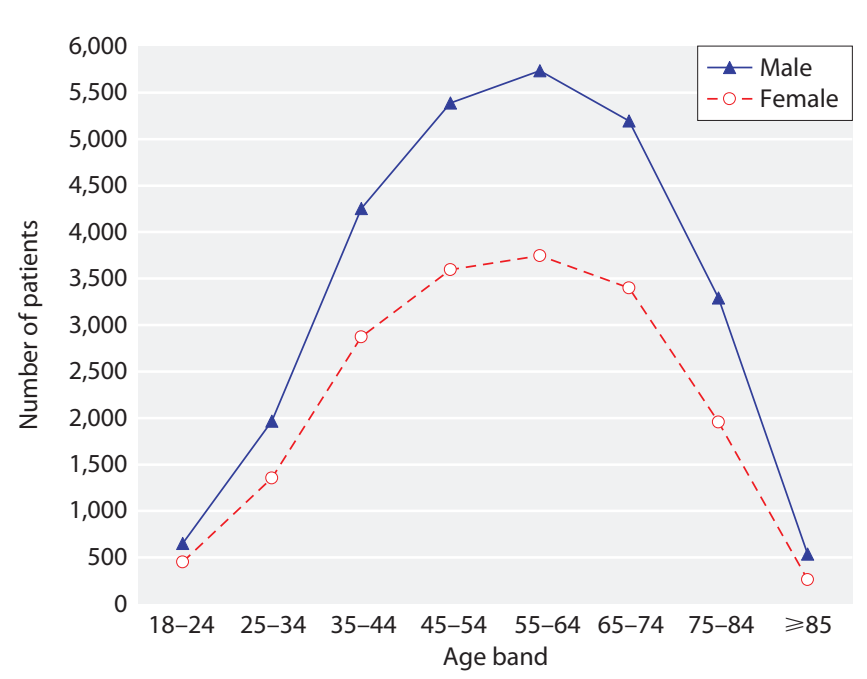

Fig. 4.9. Age profile of prevalent RRT patients by gender on 31/12/07

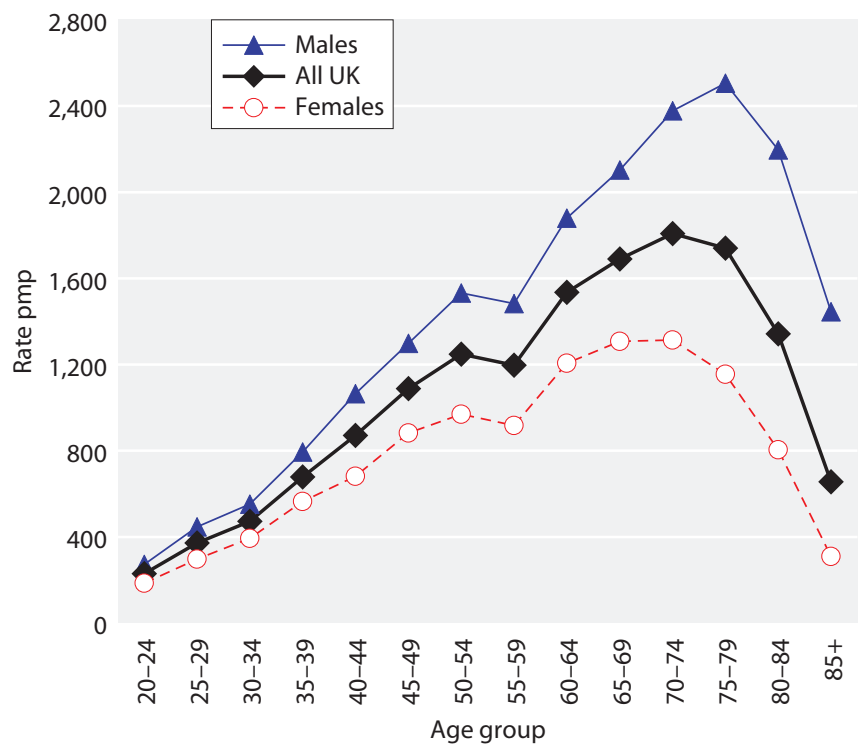

Fig. 4.10. Prevalence rate of RRT patients per million population by age and gender on $31 / 12 / 07$

though there was a high level of missing data in Scotland (where ethnicity is not a mandated item).

Among the centres with more than 50\% returns, there was wide variation between centres with respect to the proportion of patients from ethnic minorities, ranging from 0 in 4 centres (Antrim, Newry, Tyrone and Ulster) to over $50 \%$ in London West and London

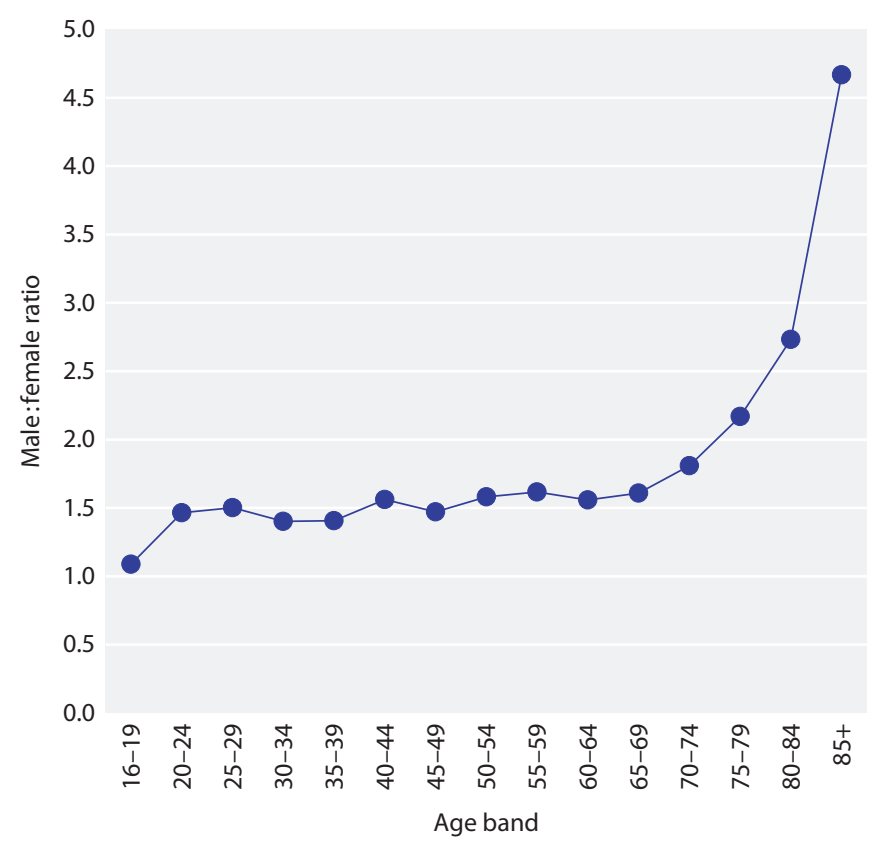

Fig. 4.11. Male:female ratio in UK RRT patients by age-band on 31/12/07 
Table 4.9. Ethnicity* of prevalent RRT patients by renal centre on 31/12/07

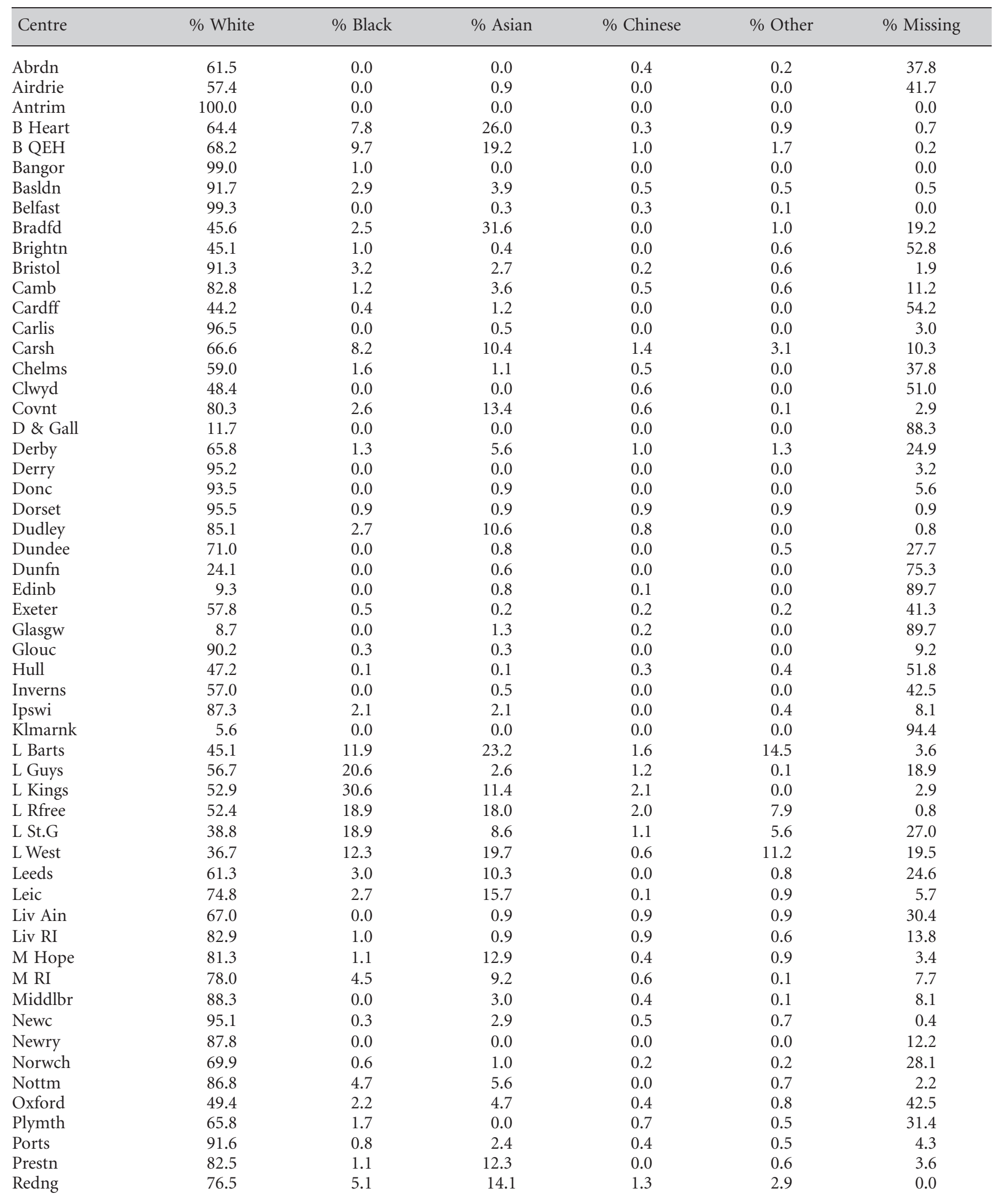


Table 4.9. Continued

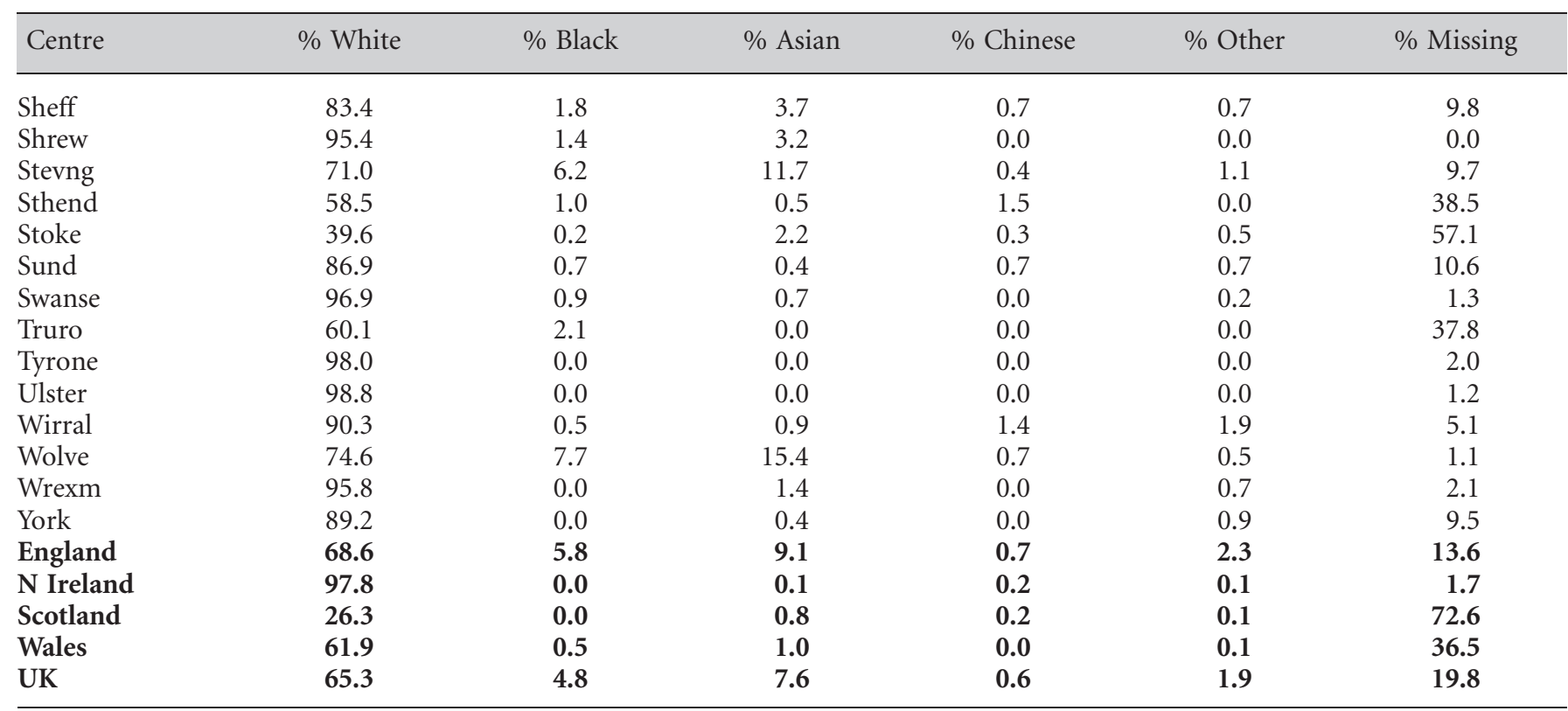

* appendix G ethnicity coding

Barts. Larger centres (quartiles by RRT population) had a larger proportion of patients from ethnic minorities (Q1 1.0\%, Q2 3.7\%, Q3 14.4\%, Q4 18.7\%). In addition centres with an ethnic minority population greater than $10 \%$ had higher numbers of patients on RRT (median 855 vs. $285: \mathrm{p}<0.001$ ), on dialysis ( 489 vs. 180 : $\mathrm{p}<0.001$ ), and with functioning transplants (839 vs. 116: $\mathrm{p}<0.001)$. Sixty percent of transplanting centres had an ethnic minority population greater than $10 \%$ compared with $28 \%$ of non-transplanting centres $(\mathrm{p}=0.015)$.

\section{Primary renal disease}

Biopsy-proven glomerulonephritis $\quad(15.3 \%$ of patients) remained the most common specific primary renal diagnosis in the 2007 prevalent cohort (table $4.10)$. The proportion with diabetes $(13.2 \%)$ was similar to 2006 [2]. The pattern was similar when the analysis was restricted to younger patients (age $<65$ years). However, in older patients the order was reversed (diabetes $15.1 \%$ vs. glomerulonephritis $8.3 \%$ ). There were other age-related differences, notably higher prevalence of the aetiology uncertain/glomerulonephritis - not biopsy proven category $(26.6 \%$ vs. $19.2 \%)$ and renovascular disease ( 8.2 vs. $1.1 \%$ ) in the older age group.

The male:female ratio was significantly greater than unity for most primary renal diseases. The gender imbalance may be influenced by the presence of factors, such as hypertension, atheroma and renovascular disease, which were more common in males, more

Table 4.10. Primary renal disease in prevalent RRT patients by age and gender on 31/12/07

\begin{tabular}{|c|c|c|c|c|c|}
\hline Primary diagnosis* & $\%$ all patients & Inter-centre range $\%$ & $\%$ age $<65$ & $\%$ age $\geqslant 65$ & $\mathrm{M}: \mathrm{F}$ ratio \\
\hline Aetiology uncertain/GN (not biopsy proven) ${ }^{* *}$ & 21.6 & $2.1-84.3$ & 19.2 & 26.6 & 1.6 \\
\hline GN (biopsy proven) ${ }^{* *}$ & 15.3 & $2.3-22.4$ & 17.8 & 10.0 & 2.2 \\
\hline Diabetes & 13.2 & $2.8-26.0$ & 12.3 & 15.1 & 1.6 \\
\hline Polycystic kidney & 9.2 & $2.0-15.8$ & 9.6 & 8.3 & 1.1 \\
\hline Hypertension & 5.4 & $1.0-16.0$ & 4.6 & 6.9 & 2.4 \\
\hline Not sent & 5.5 & $0.1-46.2$ & 5.7 & 5.2 & 1.5 \\
\hline
\end{tabular}

* appendix G ERA-EDTA coding

** GN = Glomerulonephritis 
Table 4.11. Transplant: dialysis ratios by age and primary renal disease in the prevalent RRT population on 31/12/07

\begin{tabular}{lcc}
\hline & \multicolumn{2}{c}{ Transplant: dialysis ratio } \\
\cline { 2 - 3 } Primary diagnosis & $<65$ & $\geqslant 65$ \\
\hline Aetiology uncertain/GN & & \\
(not biopsy proven) $^{*}$ & 1.4 & 0.3 \\
GN (biopsy proven) & 1.8 & 0.5 \\
Pyelonephritis & 2.1 & 0.3 \\
Diabetes & 0.7 & 0.1 \\
Polycystic kidney & 1.7 & 1.1 \\
Hypertension & 1.1 & 0.3 \\
Renal vascular disease & 0.6 & 0.1 \\
Other & 1.4 & 0.3 \\
Not sent & 1.6 & 0.3 \\
\hline
\end{tabular}

${ }^{*} \mathrm{GN}=$ Glomerulonephritis

common with increasing age and which may increase the rate of progression of kidney failure. As would be expected from the mode of inheritance, adult polycystic kidney disease (APKD) was a major exception, the ratio approximating unity in this condition. In pyelonephritis the ratio also approximated to unity, but whilst in APKD, the ratio also approximated unity in the incident cohort, in pyelonephritis the ratio was somewhat lower in the prevalent cohort than in the incident cohort (1.5). This possibly reflects poorer survival on RRT of males with this diagnosis.

Primary renal diagnosis also influenced the distribution of patients between the modalities (table 4.11), particularly the likelihood of having a functioning renal transplant. In younger patients (aged less than 65), the ratios of prevalent patients with functioning transplants to those on dialysis were higher in the groups with pyelonephritis (2.1), polycystic kidney disease (1.7) and glomerulonephritis (1.8) than in the groups with diabetes (0.7) and renovascular disease (0.6), suggesting a much higher transplant rate in the former groups. In older patients the transplant rate was generally much lower. This was reflected in the lower transplant:dialysis ratios in this group, especially those for diabetes $(0.1)$
Table 4.12. Median age, gender ratio and treatment modality in diabetic and non-diabetic prevalent RRT patients

\begin{tabular}{lcc}
\hline & All diabetes & Non-diabetics \\
\hline Number & 5,906 & 36,279 \\
M:F ratio & 1.58 & 1.52 \\
Median age on 31/12/07 & 60 & 57 \\
Median age at start of RRT & 55 & 47 \\
Median years on RRT & 2.8 & 6.1 \\
\% HD & 59 & 41 \\
\% PD & 13 & 10 \\
\% transplant & 28 & 49 \\
\hline
\end{tabular}

and renovascular disease (0.1). The exception was APKD with a ratio of 1.1 .

\section{Diabetes}

Again in 2007 there was no differentiation between Type 1 and Type 2 diabetes, since the distinction was not made in data submitted by centres in Scotland and some in Northern Ireland. The number of patients with diabetes in the 2007 prevalent cohort has increased to 5,906 , representing $14 \%$ of all patients (table 4.12). The median age at dialysis initiation was much higher in diabetics (55 vs. 47 years), though the disparity was much less in the prevalent diabetic population (60 vs. 57 years), suggestive of reduced survival in patients with diabetes. Consistent with this, the RRT vintage of prevalent patients with diabetes (2.8 years) was much less than that of prevalent without (6.1 years). The percentage of patients with a functioning transplant was much lower in prevalent diabetics than in prevalent non-diabetics $(28.1 \%$ vs. $49.4 \%)$. The contrasts were even more stark in older age patients (table 4.13), with only $6.6 \%$ of prevalent patients with diabetes having a functioning transplant compared to $24.5 \%$ of nondiabetic peers.

\section{Modalities of treatment}

The most common treatment modality in the 2007 UK prevalent cohort was transplantation (46.6\%),

Table 4.13. Age relationships by type of diabetes and modality in prevalent RRT patients $31 / 12 / 07$

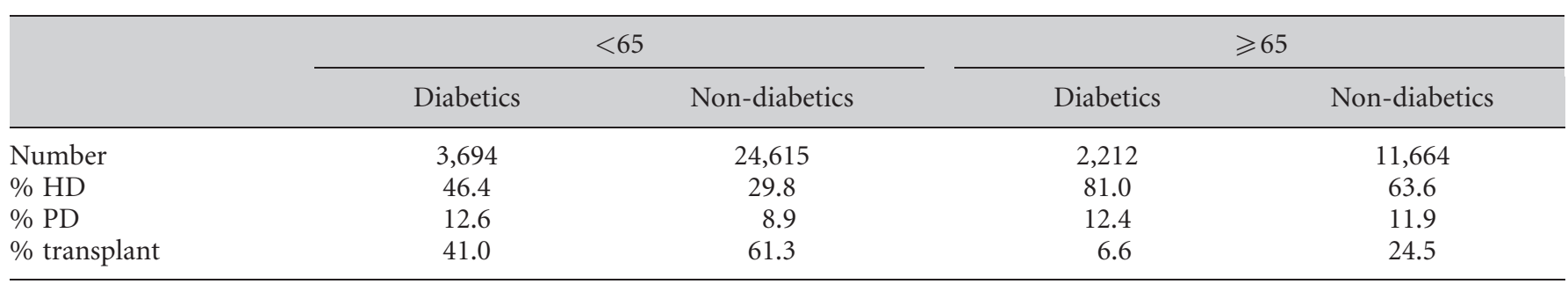




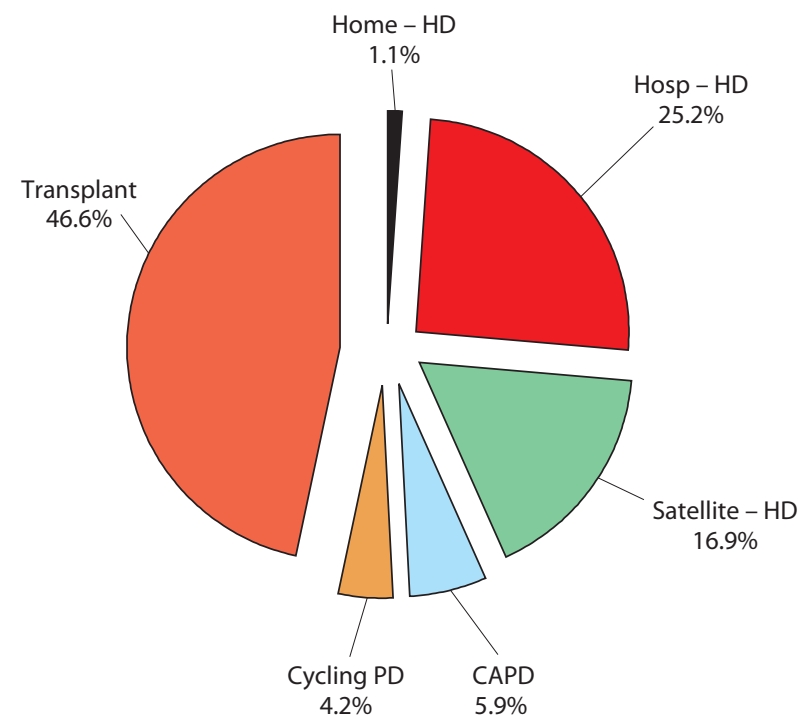

Fig. 4.12. Treatment modality in prevalent RRT patients on $31 / 12 / 07$

closely followed by centre-based HD (42.1\%) in either primary centre $(25.2 \%)$ or satellite centre $(16.9 \%)$ as depicted in figure 4.11. PD modalities made up 10.1\% of the prevalent cohort, with CAPD accounting for $5.9 \%$ and cycling PD (automated PD) for $4.2 \%$. The proportion of patients recorded as receiving CAPD using non-disconnect systems was very small, so in this analysis, has not been distinguished from those using disconnect systems. The term CAPD has been used to cover both.

Figure 4.12 shows the treatment modality in prevalent RRT patients on $31 / 12 / 2007$. Transplantation (58.8\%) was also the principal modality in patients aged less than 65, though HD (66.4\%) predominated in older patients (tables 4.14 and 4.15). A slightly higher proportion of prevalent patients over 65 were on PD compared with the younger cohort (11.9\% vs. 9.4\%). There were differences among the 4 UK countries with respect to the proportion of patients on PD according to age
Table 4.15. Percentage of prevalent dialysis patients on haemodialysis by age and UK country on $31 / 12 / 07$

\begin{tabular}{lccc}
\hline & $<65$ years & $\geqslant 65$ years & All \\
\hline England & 78 & 84 & 81 \\
N Ireland & 80 & 93 & 87 \\
Scotland & 77 & 88 & 82 \\
Wales & 70 & 81 & 76 \\
UK & $\mathbf{7 7}$ & $\mathbf{8 5}$ & $\mathbf{8 1}$ \\
\hline
\end{tabular}

group. In England and Wales, PD prevalence was higher in older patients, whilst in Northern Ireland, the reverse was the case. PD prevalence in both age groups was higher in Wales.

In general in the prevalent RRT population, age was a major factor in modality distribution (figure 4.13). With increasing age, transplant prevalence reduced, certainly beyond the age of 60 or so, whilst HD prevalence increased. The proportion of each age group treated by PD remained fairly stable across the age spectrum.

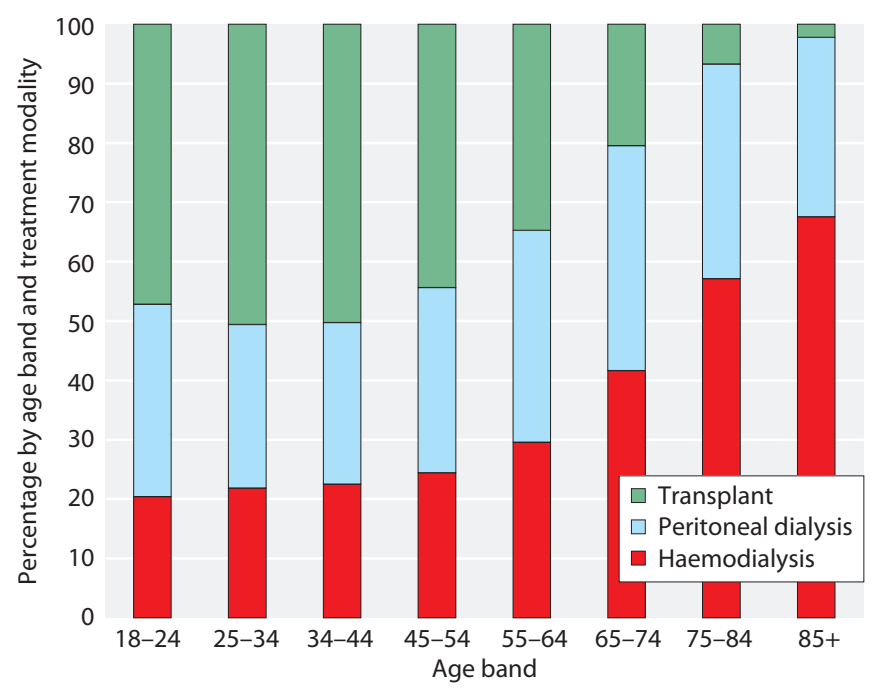

Fig. 4.13. Treatment modality distribution by age in prevalent RRT patients on $31 / 12 / 07$

Table 4.14. Treatment modalities by age in UK countries on $31 / 12 / 07$

\begin{tabular}{|c|c|c|c|c|c|c|}
\hline UK country & \multicolumn{3}{|c|}{$<65$ years } & \multicolumn{3}{|c|}{$\geqslant 65$ years } \\
\hline N Ireland & 35.0 & 8.7 & 56.3 & 74.5 & 5.6 & 19.9 \\
\hline Scotland & 32.8 & 9.9 & 57.3 & 68.4 & 9.6 & 21.9 \\
\hline Wales & 29.1 & 12.5 & 58.4 & 65.9 & 15.9 & 18.2 \\
\hline
\end{tabular}




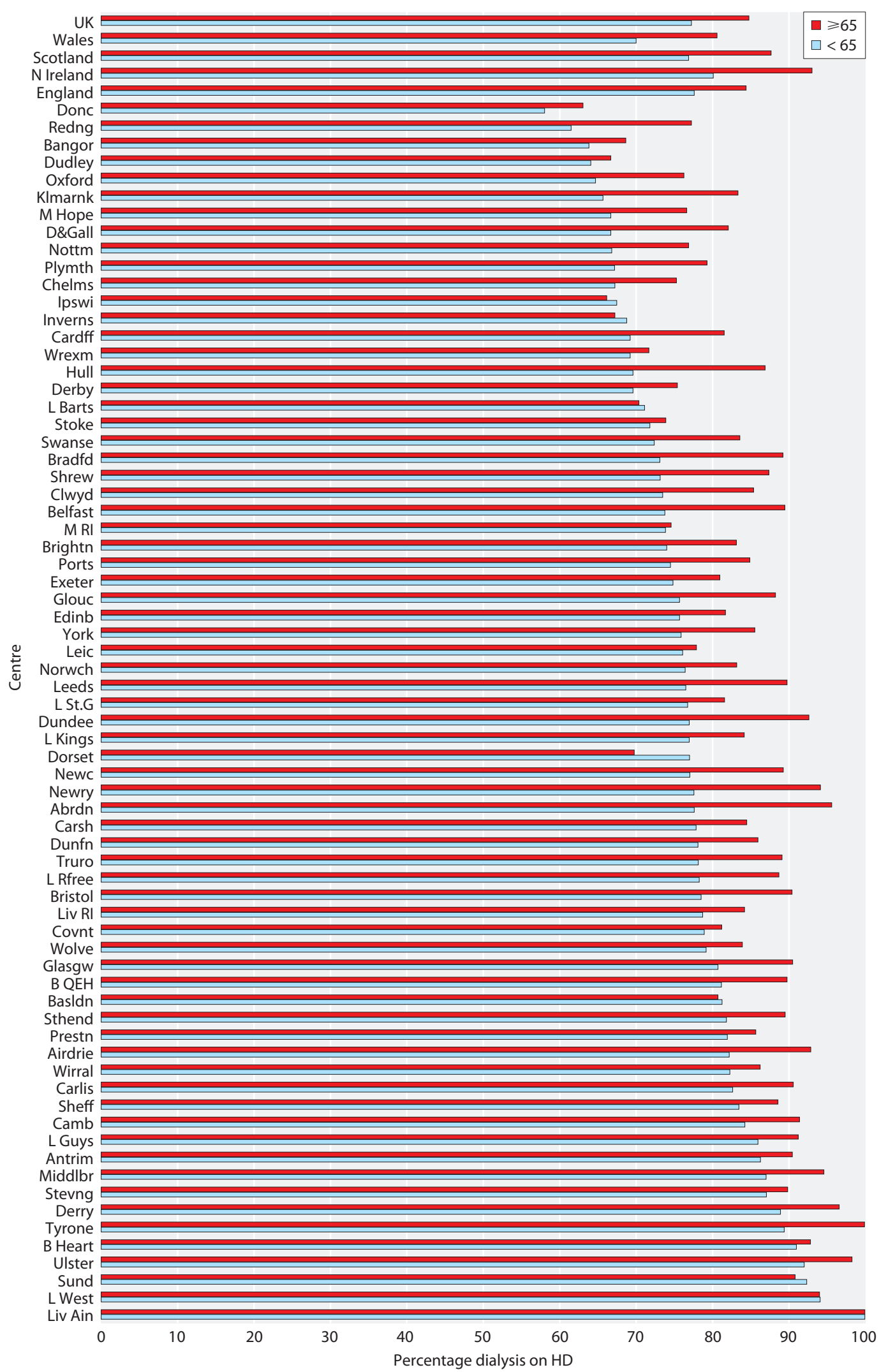

Fig. 4.14. Percentage of prevalent dialysis patients on haemodialysis by age and centre 31/12/07 
The proportion of prevalent dialysis patients on HD in the UK in the 2007 cohort was $81 \%$, and continued to increase having been $71 \%$ in 2002 . The proportion was higher still in those aged over 65 years than in younger patients ( $85 \%$ vs. $77 \%)$. There was some variation among the 4 home countries with Wales tending to have a slightly lower percentage of patients on HD and Northern Ireland slightly higher.

The proportion of prevalent dialysis patients receiving HD, ranged from $60 \%$ in Doncaster to $100 \%$ in Liverpool Aintree. In only 6 centres was the national pattern of a higher percentage of older dialysis patients receiving
HD reversed, and then only marginally. The centres were (figure 4.14), Dorset, Inverness, Ipswich, Sunderland, London Barts and Basildon.

\section{Home haemodialysis}

The percentage of dialysis patients receiving home HD varied from 0 in 20 centres, to greater than $5 \%$ of all dialysis activity in 6 centres, Sheffield (5.2\%), London Guys (5.1\%), Brighton (5.5\%), Bangor (5.1\%), Bristol (5.5\%) and Manchester Royal Infirmary (8.6\%) (table 4.16).

There was a peak in home haemodialysis use in 1982, when $60 \%$ of HD patients were on home HD

Table 4.16. Percentage of prevalent dialysis patients by dialysis modality by centre on $31 / 12 / 07$

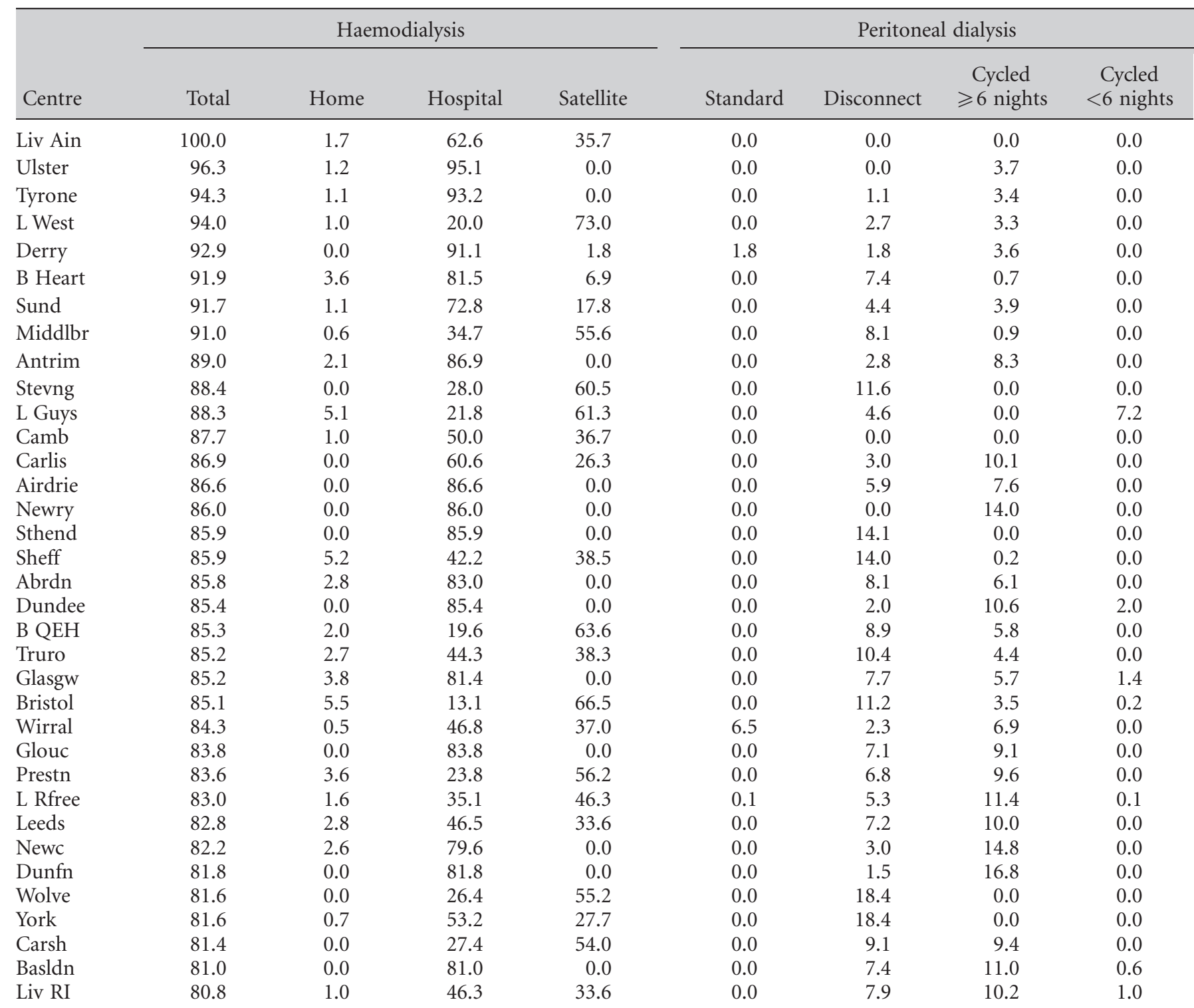


Table 4.16. Continued

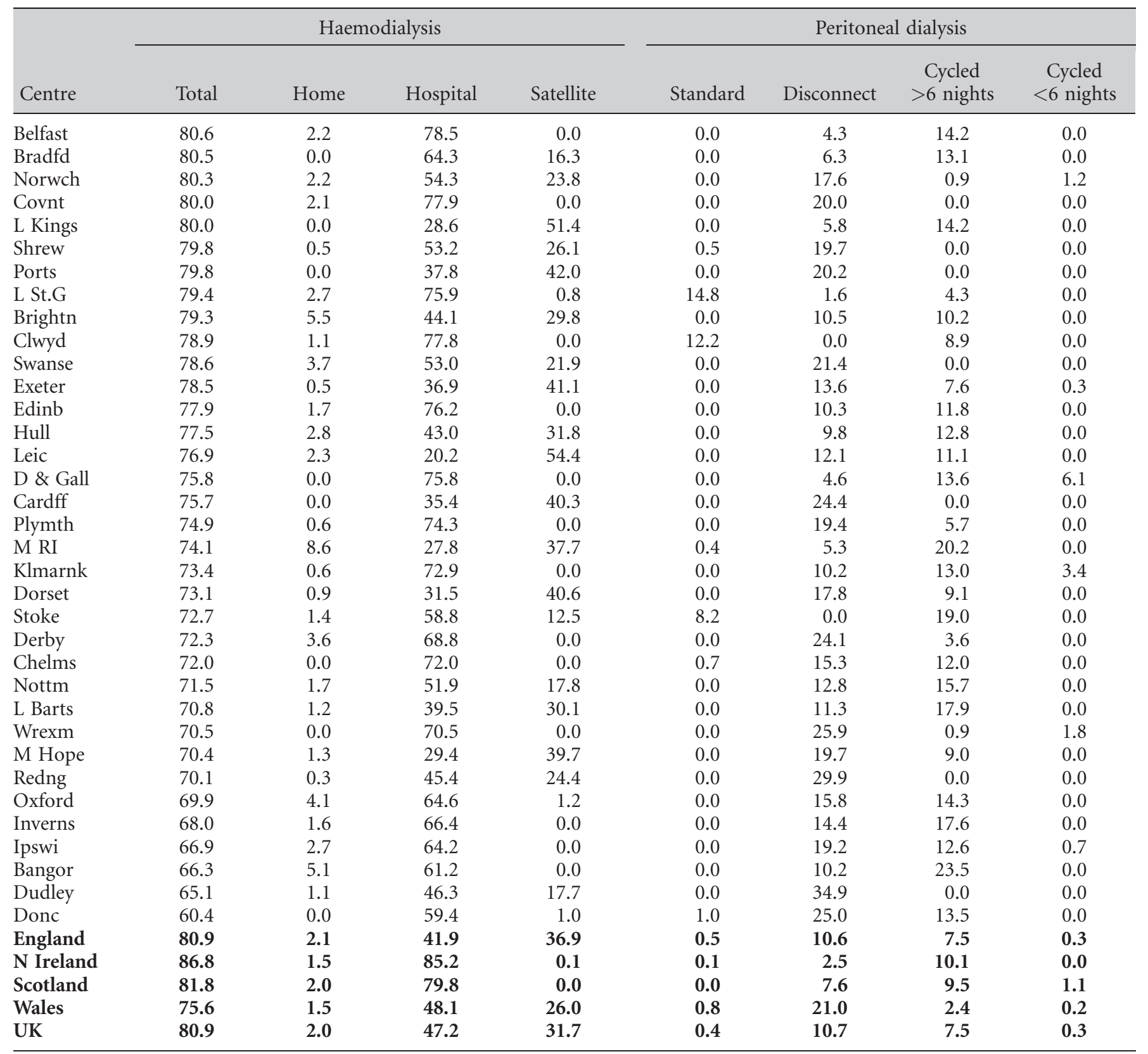

(about 2,200 patients). With an increase in the HD programme size, number of renal centres and provision of satellite HD there has been a continued fall in numbers of patients on home HD until 2003 when numbers plateaued. By 2003 only 430 patients were on home HD, about 450 from 2004 to 2006 and in 2007 this had risen slightly to 478, which accounted for $2.4 \%$ of the HD patient population. The recent increase in pre-emptive transplantation and live donation rates will also have had an impact on the numbers of patients who would be suitable for a home HD programme.

Apart from the Manchester centre (which reported to the UKRR for the first time), there was little evidence of any substantial increase in home HD activity despite NICE guidance, particularly among centres starting from zero activity in this area. Of those centres with a zero return for home haemodialysis in 2006, only Liverpool Aintree and Reading submitted non-zero returns in 2007 [2]. 


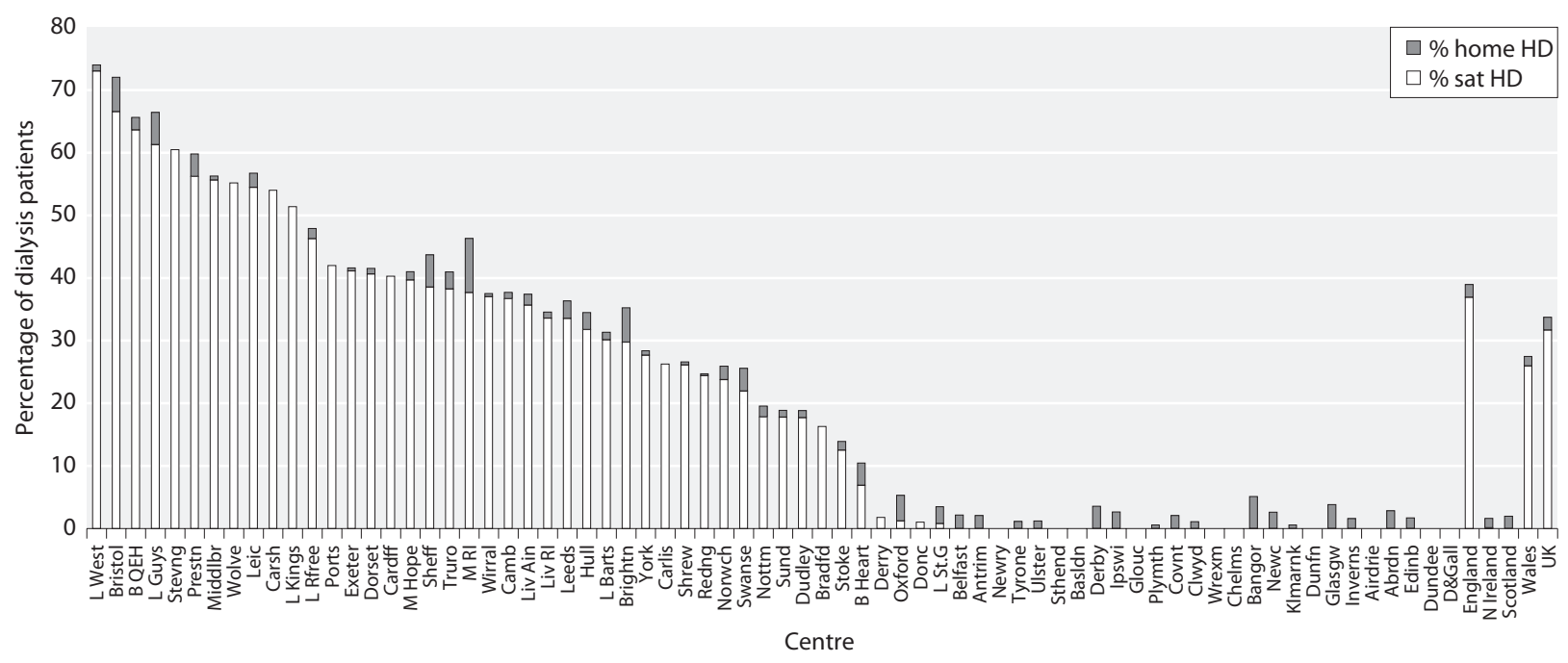

Fig. 4.15. Percentage of prevalent haemodialysis patients treated with satellite or home haemodialysis by centre in 2007

\section{Satellite Dialysis}

Twenty-six centres had no satellite haemodialysis whilst in 11 centres more than $50 \%$ of their dialysis activity took place in satellites (table 4.16). These variations with respect to home and satellite haemodialysis are depicted in figure 4.15. There was also much diversity between centres in the proportion of PD patients on cycling treatments, ranging from 0 to $100 \%$ (table 4.16). Eleven of the 68 centres with a PD programme, had no patients on cycling $\mathrm{PD}$, whilst in two centres (Ulster and Newry) all PD patients were on this form of the modality.

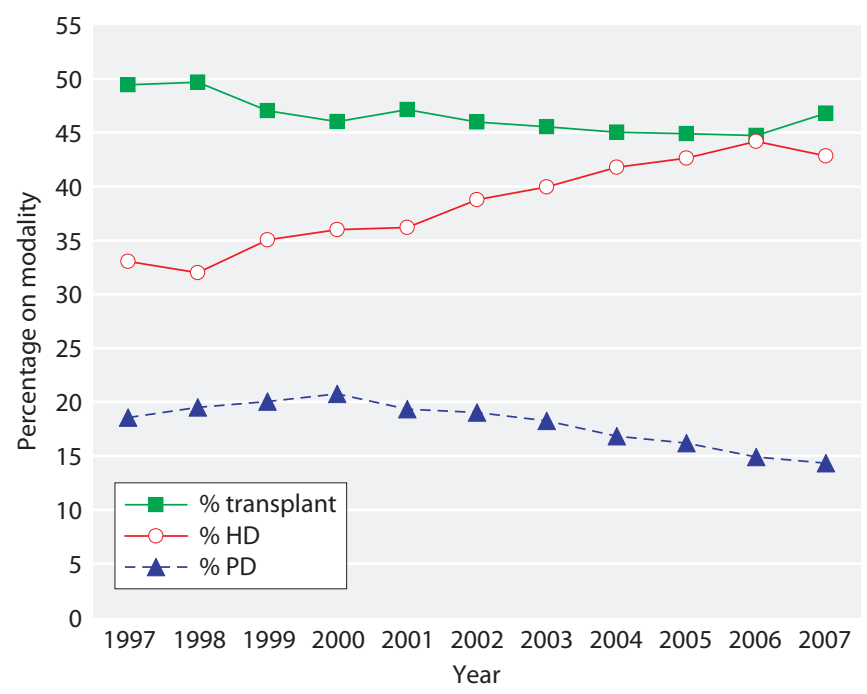

Fig. 4.16. Modality changes in prevalent RRT patients from 1997-2007 (England and Wales)

\section{Change in modality}

The relative proportion of RRT modalities in prevalent patients has changed dramatically over the past decade. The main features are depicted in figure 4.16, which describes a sustained decrease in the proportion of patients treated by PD. By way of compensation there has been a continuous rise in the proportion of patients treated by HD. The proportion with a functioning transplant has fallen slightly over the same period.

Figure 4.17 depicts in more detail the changes in the prevalent dialysis population during this time and highlights a sustained reduction in the proportion of these patients treated by PD. This change was almost

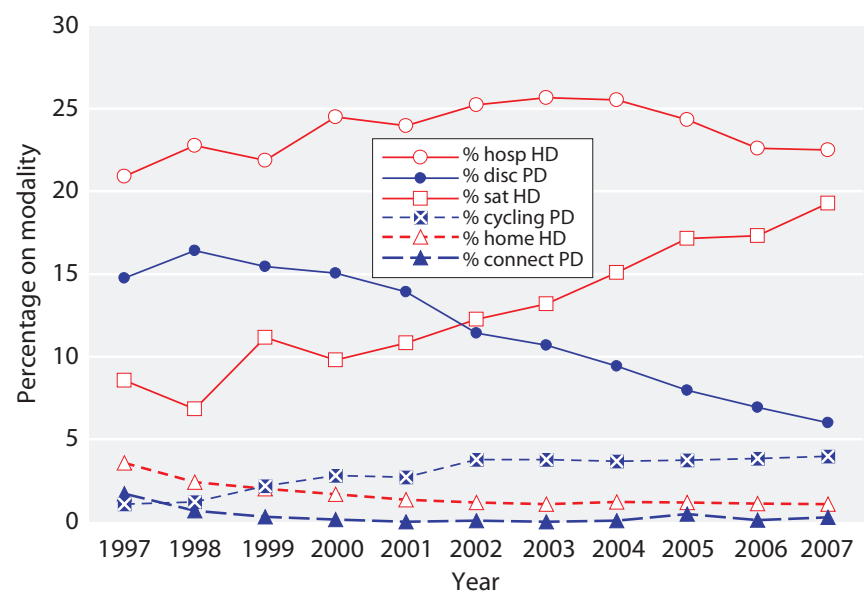

Fig. 4.17. Detailed dialysis modality changes in prevalent RRT patients from 1997-2007 (England and Wales) 
completely counterbalanced by growth in the proportion of prevalent HD patients treated at satellite centres. The hospital haemodialysis population, other than the proportion dialysing in satellite centres has remained fairly constant.

Conflict of interest: none

\section{References}

1. Office for National Statistics. www.statistics.gov/census2001

2. Ansell D, Feehally J, Feest TG, Tomson C, Williams AJ, Warwick G. UK Renal Registry report 2007. UK Renal Registry Bristol; Chapter 4:p 49-74 